\title{
Gut microbiota modulates adoptive cell therapy via CD8 $\alpha$ dendritic cells and IL-12
}

\author{
Mireia Uribe-Herranz, ${ }^{1,2}$ Kyle Bittinger, ${ }^{3}$ Stavros Rafail, ${ }^{1}$ Sonia Guedan, ${ }^{4}$ Stefano Pierini, ${ }^{1,2}$ \\ Ceylan Tanes, ${ }^{3}$ Alex Ganetsky, ${ }^{5}$ Mark A. Morgan, ${ }^{1}$ Saar Gill, ${ }^{6}$ Janos L. Tanyi, ${ }^{1}$ Frederic D. Bushman, ${ }^{7}$ \\ Carl H. June, ${ }^{4}$ and Andrea Facciabene ${ }^{1,2}$ \\ 'Ovarian Cancer Research Center, and ${ }^{2}$ Department of Radiation Oncology, Perelman School of Medicine, University of \\ Pennsylvania, Philadelphia, Pennsylvania, USA. ${ }^{3}$ Division of Gastroenterology, Hepatology, and Nutrition, Children's \\ Hospital of Philadelphia, Philadelphia, Pennsylvania, USA. ${ }^{4}$ Abramson Cancer Center and the Department of Pathology \\ and Laboratory Medicine, Perelman School of Medicine, ${ }^{5}$ Department of Pharmacy, ${ }^{6}$ Division of Hematology Oncology, \\ Department of Medicine, and 'Department of Microbiology, Perelman School of Medicine, University of Pennsylvania, \\ Philadelphia, Pennsylvania, USA.
}

Adoptive T cell therapy (ACT) is a promising new modality for malignancies. Here, we report that adoptive T cell efficacy in tumor-bearing mice is significantly affected by differences in the native composition of the gut microbiome or treatment with antibiotics, or by heterologous fecal transfer. Depletion of bacteria with vancomycin decreased the rate of tumor growth in mice from The Jackson Laboratory receiving ACT, whereas treatment with neomycin and metronidazole had no effect, indicating the role of specific bacteria in host response. Vancomycin treatment induced an increase in systemic CD8 $\alpha^{+} D C s$, which sustained systemic adoptively transferred antitumor T cells in an IL-12-dependent manner. In subjects undergoing allogeneic hematopoietic cell transplantation, we found that oral vancomycin also increased IL-12 levels. Collectively, our findings demonstrate an important role played by the gut microbiota in the antitumor effectiveness of ACT and suggest potentially new avenues to improve response to $\mathrm{ACT}$ by altering the gut microbiota.

Conflict of interest: The authors have declared that no conflict of interest exists.

Submitted: May 12, 2017

Accepted: January 18, 2018

Published: February 22, 2018

Reference information: JCI Insight. 2018;3(4):e94952. https:// doi.org/10.1172/jici.nsight.94952.

\section{Introduction}

Infiltration of primary tumors by Th1 and cytotoxic T cells provides one of the strongest prognostic factors for outcome at all stages of disease $(1,2)$. The importance of $\mathrm{T}$ cells for the antitumor response has been recently demonstrated using adoptive transfer of in vitro expanded tumor infiltrating lymphocytes (TIL) or genetically modified T cells. These approaches have shown enormous promise in clinical trials, including overall response rates of $40 \%$ in metastatic melanoma, $90 \%$ in acute lymphoblastic leukemia, and $40 \%$ in chronic lymphoblastic leukemia (3-9). As an alternative strategy to generate autologous antitumor T cells, expansion of tumor-specific T cells from peripheral blood is an attractive approach. A phase I study that evaluated the feasibility of expanding HER-2/neu (HER2) vaccine-primed peripheral blood T cells ex vivo resulted in clinical responses in $43 \%$ of patients (10). Moreover, several studies have shown that patients can be immunized against tumor-associated antigens (TAA) (11-17), expanding the chances to implement this method. In mice, $\mathrm{T}$ cells purified and expanded from periphery after vaccination with TAA enhanced the effects of therapeutic vaccines in weakly immunogenic tumor models (18) or in a B16 melanoma model after a BM transplant (19).

Despite the promising response rates of these approaches, there is still an incidence of treatment failure. The reasons for this are incompletely understood (20) but likely include endogenous barriers such as peripheral tolerance and immune evasion by the tumor. The limited success also raises the question of other, yet to be discovered, regulators of $\mathrm{T}$ cell function that are relevant to tumor control.

Several studies have shown the influence of commensal microbes on $\mathrm{T}$ cell phenotype and function (21-24). For instance, colonization of germ-free mice gut with a cocktail of bacteria from Clostridiales clusters IV, XIVa, and XVIII is sufficient to drive Treg differentiation (23). Another example is the influence of bacteria or their byproducts on tumor progression in mammary glands, a process mediated by the host immune system $(25,26)$. Moreover, the gut microbiota has been demonstrated to affect antitumor immune approaches, including immune check-point inhibition (27-29). The translocation of gut bacteria 
to the i.p. space, due to epithelial layer damage, can induce transitory infection with systemic elevation of IL-12 that affects the efficacy of ACT (30). All of these studies provide strong evidence for close and complex interplay between the gut microbiome, tumor development, and antitumor immunotherapies.

DCs are professional antigen presenting cells (APC) that orchestrate various innate and adaptive immune system effector mechanisms and, thus, play a critical role in the antitumor immune response (31-34). The phenotype and function of DCs have been demonstrated to be influenced by the gut microbiota (35).

Here, we examine the relationship between gut microbiome composition and the response to ACT. Indeed, we demonstrate that ACT efficacy is directly influenced by the gut microbiota in a mouse model of cervical cancer. Furthermore, in mice from The Jackson Laboratory (Jax), we show that ACT efficacy is improved by treatment with oral vancomycin, an antibiotic that mostly targets gram-positive bacteria in the gut. Our study indicates that the effect of vancomycin treatment is dependent on an increase of systemic $\mathrm{CD} 8 \alpha^{+} \mathrm{DCs}$ that, through IL-12 expression, sustains systemic adoptively transferred antitumor T cells.

\section{Results}

The gut microbiota influences ACT efficacy. Previous studies have demonstrated that, despite sharing the same genetic background, animals from different vendors harbor different gut microbiota (36). Therefore, we assessed the impact of the gut microbiome on ACT by comparing tumor growth in C57BL/6 female mice from Jax and Harlan Laboratories, now Envigo (Har). Because effective T cells against the E6/E7 HPV proteins can be generated in patients with HPV-associated cancers (12), we developed a model of T cell therapy based on the tissue culture number 1 (TC1) cervix and lung cancer model (37), in which HPV16 E6/E7 proteins are expressed. To study the effects of the gut microbiota on ACT, we obtained $5 \times 10^{6} \mathrm{CD}^{+} \mathrm{T}$ cells polarized under Th1 condition - as ACT efficacy depends of cytotoxic activity and IFN- $\gamma$ production (38) - that were generated by vaccination (39) of donor mice (Jax), and we transferred them to lympho-depleted TC1 tumor-bearing animals (12) 5 days after tumor implantation and 7 days after lympho depletion.

Adoptive $\mathrm{T}$ cell transfer influenced tumor progression in both sets of mice (Figure 1A). In Har mice receiving ACT, tumor growth was almost completely abrogated $(P<0.001)$, while in Jax mice, ACT was less effective $(P<0.001)$. Tumor growth differences between Har and Jax receiving ACT were significant $(P<$ 0.001). To investigate differences in gut microbiota that could be associated with the observed differences in ACT efficacy, we carried out 16S rRNA marker gene sequencing of stool samples collected at 7 and 21 days after ACT. The fecal bacterial communities from Har and Jax mice differed substantially when compared using unweighted UniFrac distance $(P<0.001$, PERMANOVA test, Figure 1B). This difference was attributable primarily to a diverse range of Bacteroidetes taxa in Har mice, namely Bacteroides, Parabacteroides, Prevotella, the Rikenellaceae family, and the candidate family Bacteroidales S24-7 (Figure 1C). The Jax mice were dominated by Bacteroidales S24-7; no other Bacteroidetes taxa were detected (Figure 1C). These vendor-specific differences in fecal microbiota were also observed in a separate set of mice from each vendor sampled upon arrival (Supplemental Figure 1; supplemental material available online with this article; https://doi.org/10.1172/jci. insight.94952DS1). The change in microbiome composition from 7-21 days within the vendor groups was significantly smaller than the difference between vendors ( $P=0.03$, PERMANOVA test).

To further investigate the relevance of the gut microbiota on ACT efficacy, we treated Har and Jax mice with different antibiotics, as early germ-free condition can have durable consequences that are not possible to change by the introduction of gut microbiota (40). We orally administered vancomycin, an antibiotic against gram-positive bacteria that is not absorbed; thus, its activity is limited to the intestine (41). Vancomycin was orally administered for 10 days before initiation of ACT (42). In Har and Jax mice receiving vancomycin, the total number of bacterial taxa was significantly decreased at 7 days following ACT $(P<0.001$, Figure 2A), due primarily to depletion of gram-positive taxa. In addition, vancomycin treatment eliminated gram-negative bacteria of the Bacteroidetes phylum, such that the phylum was completely absent in Jax mice at 7 days after ACT and only Parabacteroides remained in Har mice (Figure 2B). These taxa were absent in both the control and vancomycin groups of Jax mice. Additional taxonomic differences between treatment groups are outlined in Supplemental Figure 2. At 21 days, the similarity between vancomycin-treated mice and non-vancomycin-treated mice from the same vendor increased relative to the 7-day time point, indicating a partial transition back to the vendor-specific microbiome states upon discontinuation of vancomycin $(P<0.001$, Figure $2 \mathrm{C})$.

Following treatment with vancomycin, tumor progression was reduced in Jax mice $(P<0.001$, Figure $2 \mathrm{D})$, matching the impact of ACT in Har mice without vancomycin $(P=0.42)$. Har mice receiving vancomycin and ACT exhibited no change relative to those not receiving vancomycin $(P=0.45)$. Although 25 days was chosen 

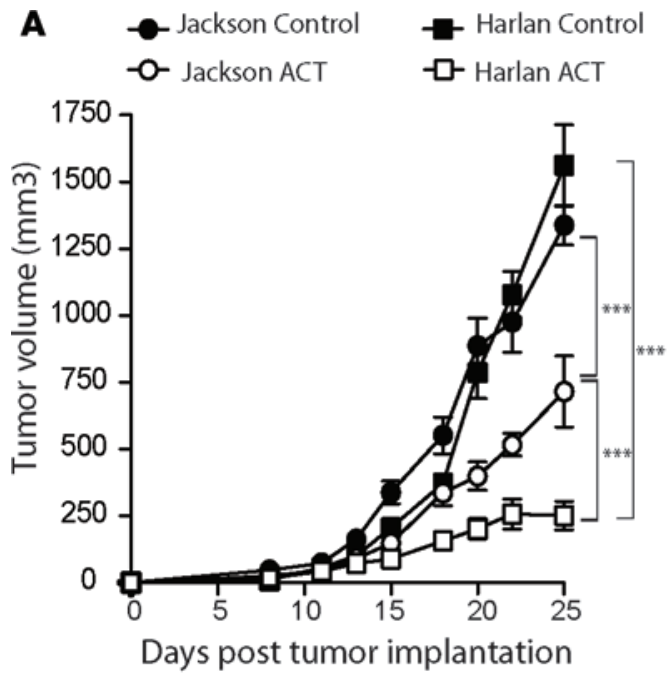

B
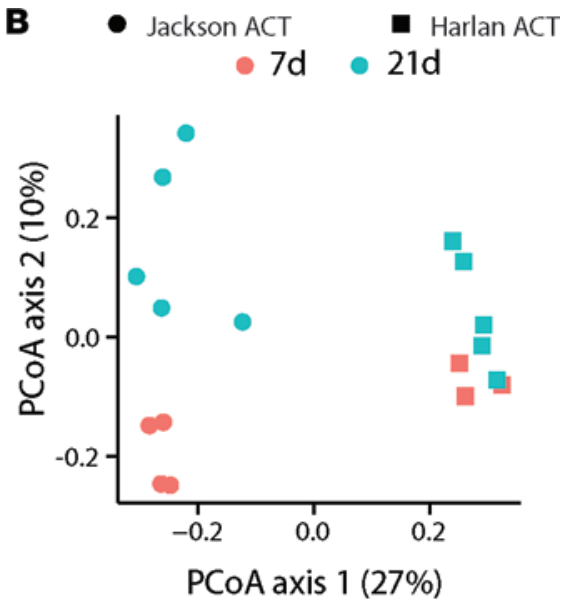

Figure 1. The gut microbiota influences effectiveness of adoptive cell therapy. (A) Impact of ACT on tumor growth in mice obtained from Jackson and Harlan. Means \pm SEM are shown. Differences in tumor volume were evaluated with linear mixed effects models. ${ }^{* *} P<0.001$. (B) Principal coordinates analysis ( $P C O A)$ of bacterial community composition, using unweighted UniFrac distance. (C) Bacterial taxa observed in Jackson and Harlan mice at 7 days and 21 days following ACT.

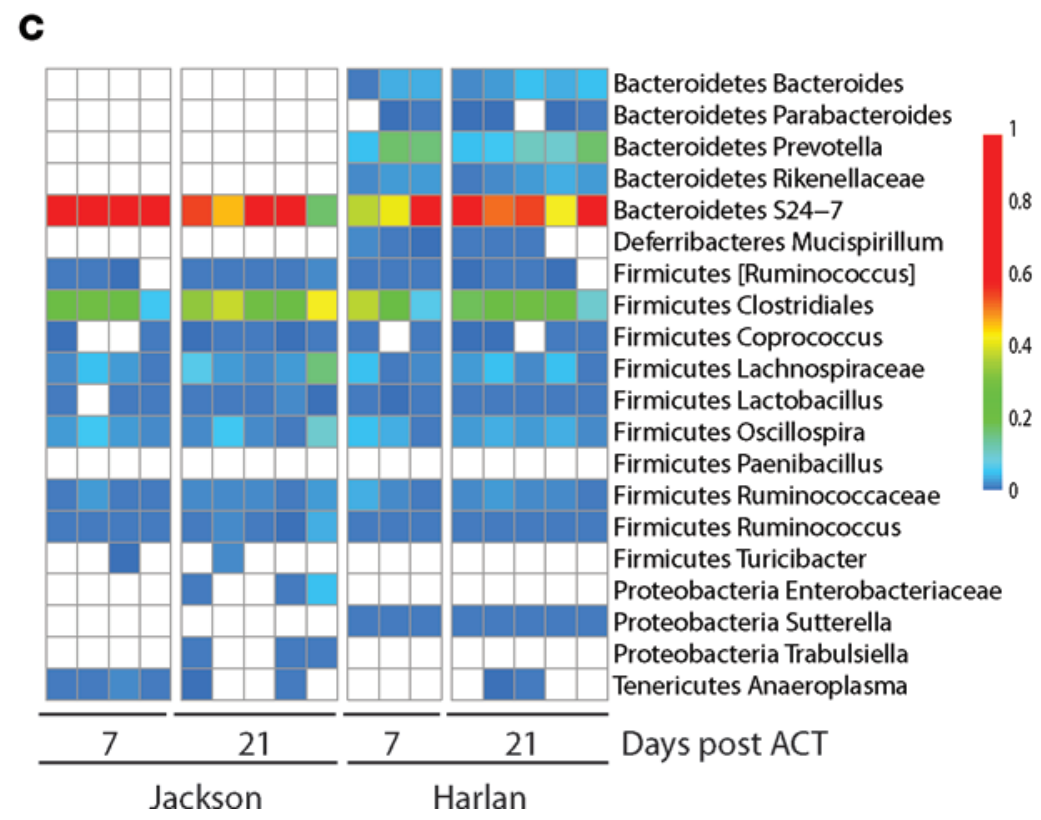

as the end point facilitating the comparison of immunologic status to controls, trends in tumor progression were consistent at 35 days (Supplemental Figure 3A). Overall survival was significantly improved, as well, with the addition of vancomycin to the ACT treatment in Jax mice (Supplemental Figure 3B). Thus, introduction of a single antibiotic was sufficient to alter tumor progression. Because vancomycin was unable to cross the epithelial barrier (41) and produced substantial alterations in the gut bacterial populations, we looked to specific changes in the gut microbiota that were associated with tumor progression. We identified taxa in the Bacteroidetes and Firmicutes phyla where the changes in abundance or presence/absence were consistent with the changes in tumor progression (Supplemental Figures 4 and 5, respectively). All taxa identified belonged to the order Clostridiales, or to the Bacteroidales S24-7 family. Taken together, these data provide strong evidence that the composition of the gut microbiota influences the efficacy of ACT in controlling tumor progression.

Efficacy of ACT is increased by vancomycin but not neomycin/metronidazole antibiotic treatment. The balance of different cohabitants in the intestine is maintained by complex interactions among the commensals and the host. The imbalances produced by vancomycin treatment affected overall gut microbiome homeostasis and induced dysbiosis among gram-positive and -negative commensals (Figure 2B). To investigate the role of gram-positive and -negative bacteria subgroups in the modulation of the immune system and the ACT outcome, Jax TC1 tumor-bearing mice were treated with either vancomycin or neomycin/metronidazole (Neo/Met), an antibiotic cocktail that targets gram-negative bacteria, prior to the adoptive transfer of $\mathrm{T}$ 
A

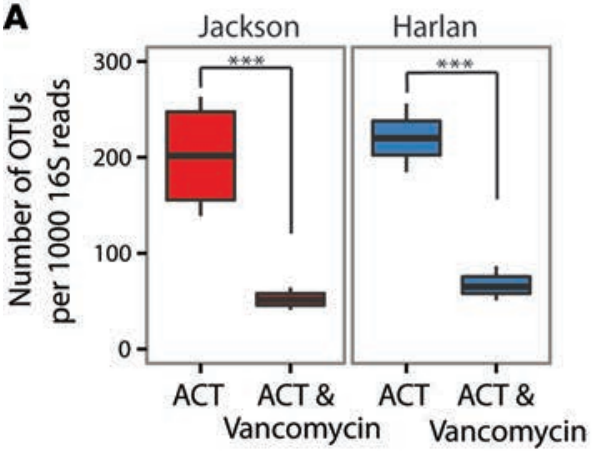

B

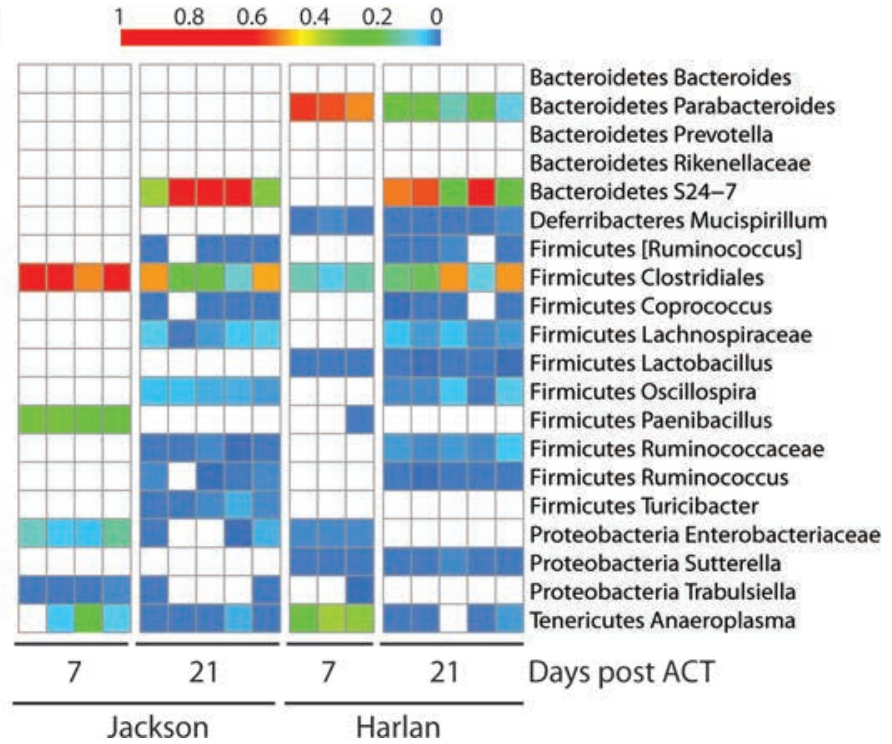

- Jackson ACT O Jackson ACT \& Vancomycin

- Harlan ACT $\square$ Harlan ACT \& Vancomycin
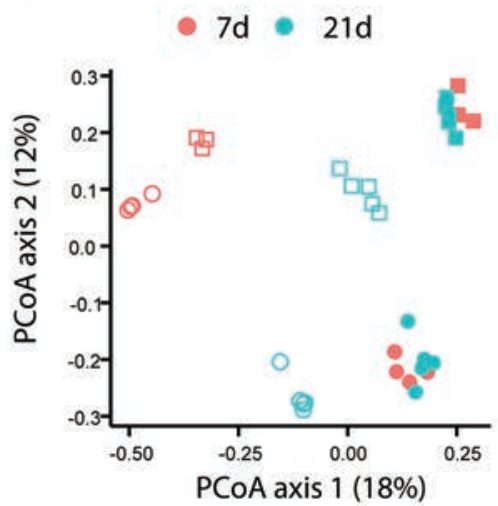

D

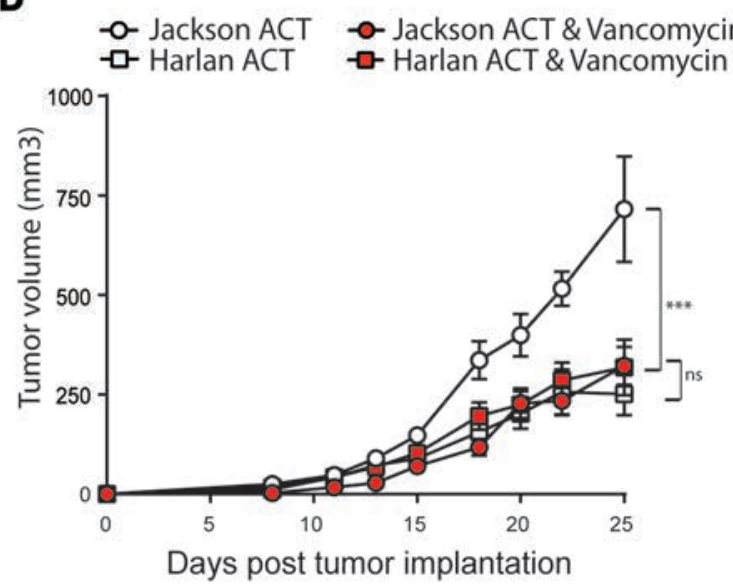

Figure 2. The modulation of gut microbiota through antibiotics impacts efficacy of adoptive cell therapy. (A) Difference in number of bacterial taxa observed after treatment with vancomycin. (B) Bacterial taxa observed and (C) community composition after treatment with vancomycin. (D) Tumor growth of mice treated in combination of ACT and vancomycin. Tumor growth data are representative of 2 individual experiments with at least 5 mice per group, means \pm SEM are shown (same control is shown in $\mathbf{A}$ ). Differences in tumor volume were evaluated with linear mixed effects models. ${ }^{*} P<0.05,{ }^{* * *} P<0.001$.

cells. Antibiotic treatment continued for 10 days, beginning 1 day before the mice were challenged. Mice treated with a combination of ACT and vancomycin (Figure 3A) showed a significant decrease in tumor growth compared with mice that received ACT only $(P<0.001)$ and with the control group $(P<0.001)$, confirming the previous data (Figure 2D). Tumor growth in mice treated with the combination ACT and Neo/Met (Figure 3B) was not statistically different from those that received ACT alone $(P=0.50)$ but significantly smaller in comparison with the control group $(P<0.001)$. We confirmed these results with a second model of ACT, where we adoptively transferred nonpolarized $\mathrm{CD}^{+} \mathrm{T}$ cells generated from vaccination. Similar to the results obtained using Th1 polarized T cells, mice treated with ACT using nonpolarized $\mathrm{T}$ cells showed a significant decrease in tumor growth compared with control mice $(P<0.01)$, and an ulterior increase of ACT efficacy was observed when vancomycin was added to ACT $(P<0.001$, Figure 3C). Likewise, tumor growth in mice treated with the combination of adoptively transferred nonpolarized $\mathrm{T}$ cells and Neo/Met did not differ from the nonpolarized ACT-only group $(P=0.73$, Figure $3 \mathrm{D})$. These results suggest that the bacteria population responsible for opposing the effect of ACT in Jax mice reside within the vancomycin-sensitive, and not the Neo/Met-sensitive, population.

Increased ACT efficacy by antibiotic treatment is phenocopied by fecal microbiota transplant. To establish a causal relationship between the gut microbiota and the efficacy of ACT, we transplanted the fecal microbiota from Jax to Har mice. We first depleted the resident gut microbiome of Har recipient mice, following a previously published antibiotic regimen $(43,44)$. We confirmed the efficacy of the antibiotic treatment by $\mathrm{PCR}$, with16S rRNA gene primers targeting either gram-positive or gram-negative bacteria (Figure 4A). 

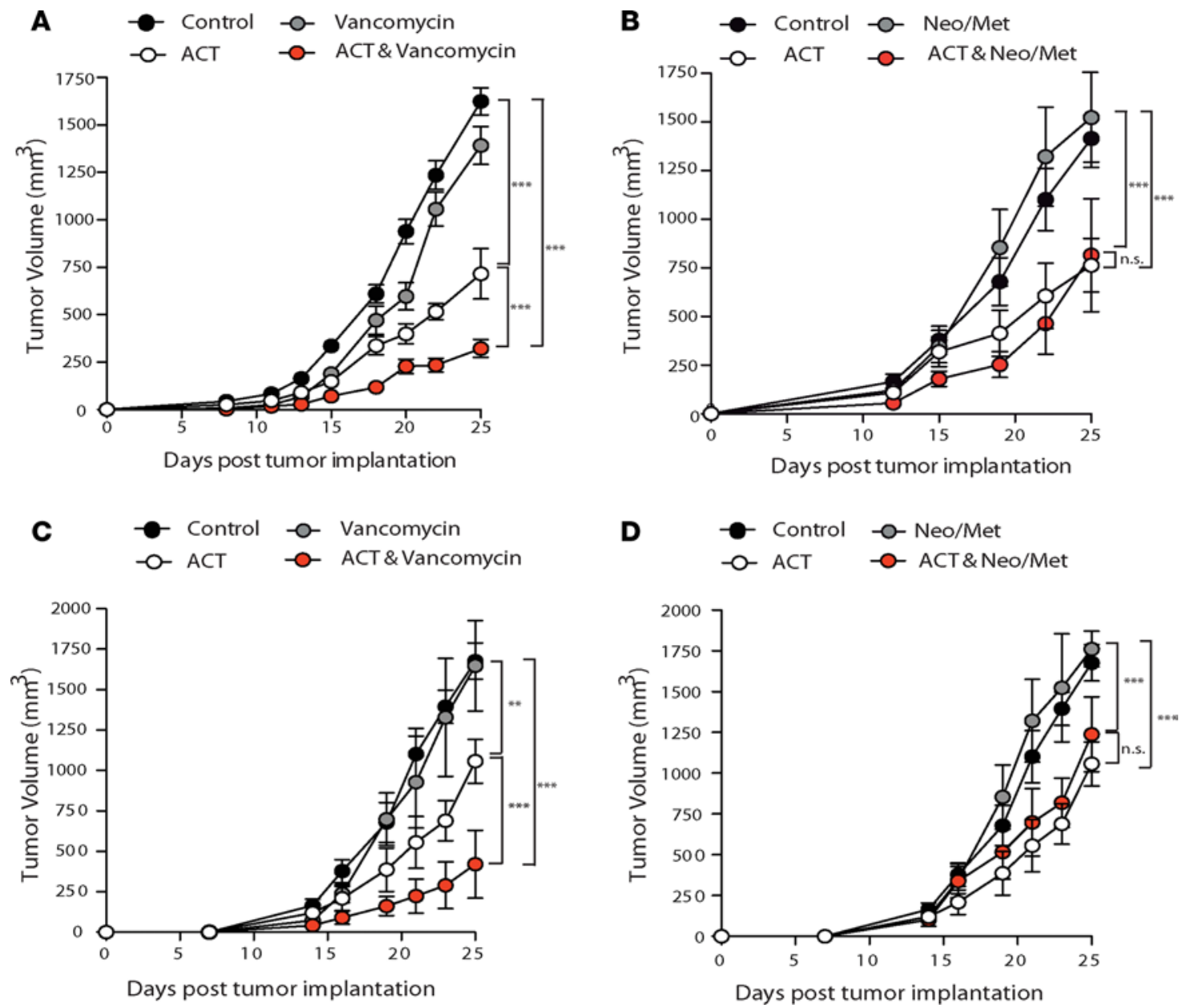

Figure 3. The efficacy of ACT is increased by vancomycin but not neomycin/metronidazole antibiotic treatment. Tumor growth of mice treated with $\mathrm{ACT}$, using $\mathrm{CD3}^{+} \mathrm{T}$ cells from immunized mice polarized under Th1 conditions, and (A) vancomycin or (B) neomycin/metronidazole (Neo/Met). Tumor growth of mice treated with ACT, using nonpolarized cells from immunized mice, and (C) vancomycin or (D) Neo/Met. Tumor growth data are representative of 3 independent experiments with 5 mice per group. Means \pm SEM are shown. Differences in tumor volume were evaluated with linear mixed effects models. ${ }^{* *} P<0.01,{ }^{* *} P<0.001$.

Har recipient mice were then gavaged twice with fecal extract obtained from Jax mice (1-2 pellets/recipient). To confirm engraftment, we assayed for the presence of Segmented Filamentous Bacteria, which are absent in Jax mice and present in Har mice (36). As expected, the bacteria were present in Har mice before antibiotic treatment and absent following engraftment from Jax mice (Figure 4B).

Two weeks after gavage, donor and recipient mice were enrolled in the ACT protocol. To track the microbiota composition following transplant, we carried out 16S rRNA marker gene sequencing of stool samples collected at 0 and 7 days after tumor implantation (Figure 4C). On day 0, 2 weeks after gavage, the microbiota composition of Har mice that received the fecal transplant differed from that of the Jax donors ( $P=0.02$, PERMANOVA test) but nonetheless had greater similarity to Jax donor mice than to native Har mice $(P<0.001,2$-tailed $t$ test of unweighted UniFrac distances between groups Figure 4D). On day 7, the distances between samples from donor and recipient mice decreased significantly relative to day $0(P<0.001$, Figure 4D). Thus, the microbiota of recipient mice adopted a similar configuration to that of the donors, and this configuration was maintained at the second time point. When vancomycin was administered to Har mice following the Jax microbiota transfer, the Har recipients displayed synergy between ACT and antibiotic treatment $(P=0.02)$, replicating the results from native Jax mice ( $P=0.39$, Figure $4 \mathrm{E})$. Thus, the microbiota-dependent response to ACT was successfully transferred between mice, confirming that extrinsic maneuvers could be applied to control the gut microbiota and improve ACT immunotherapy. 
$\mathbf{A}$

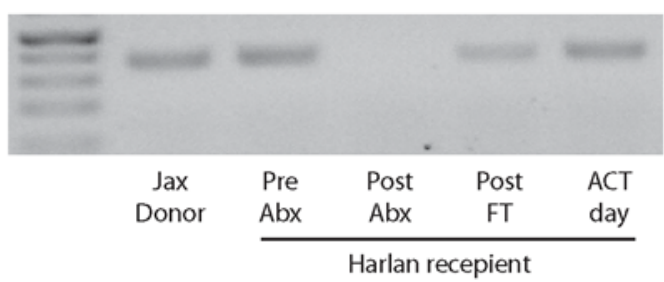

B

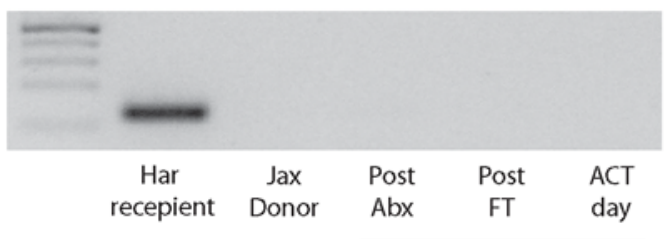

Harlan recepient

C

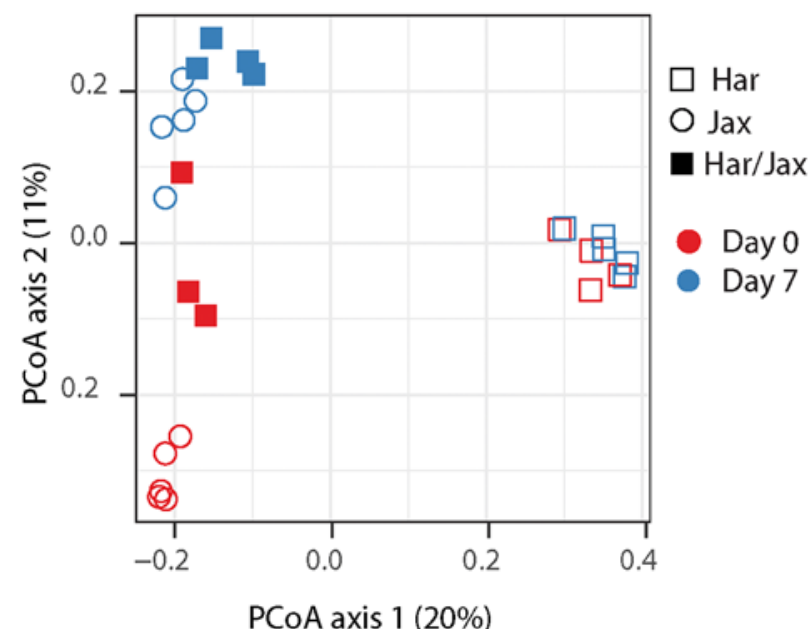

D Har/Jax ACT Vancomycin
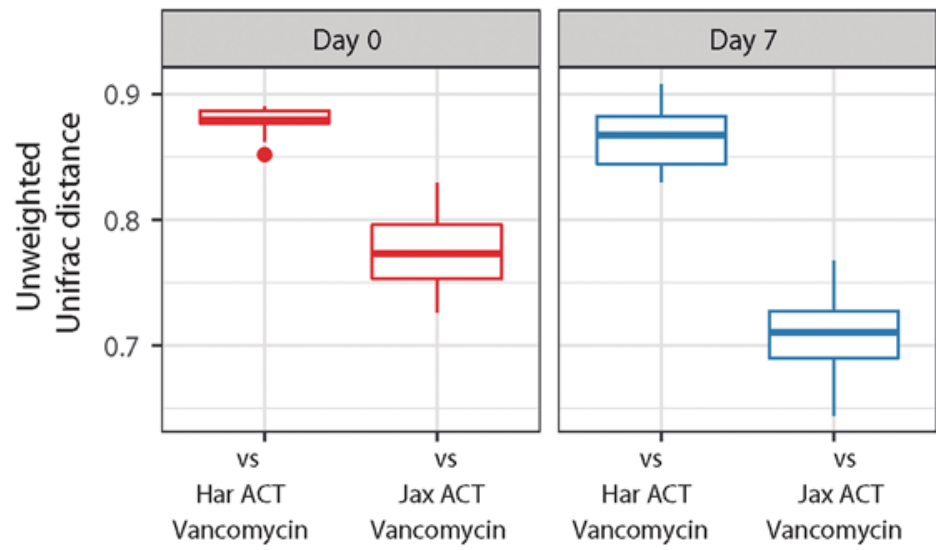

E
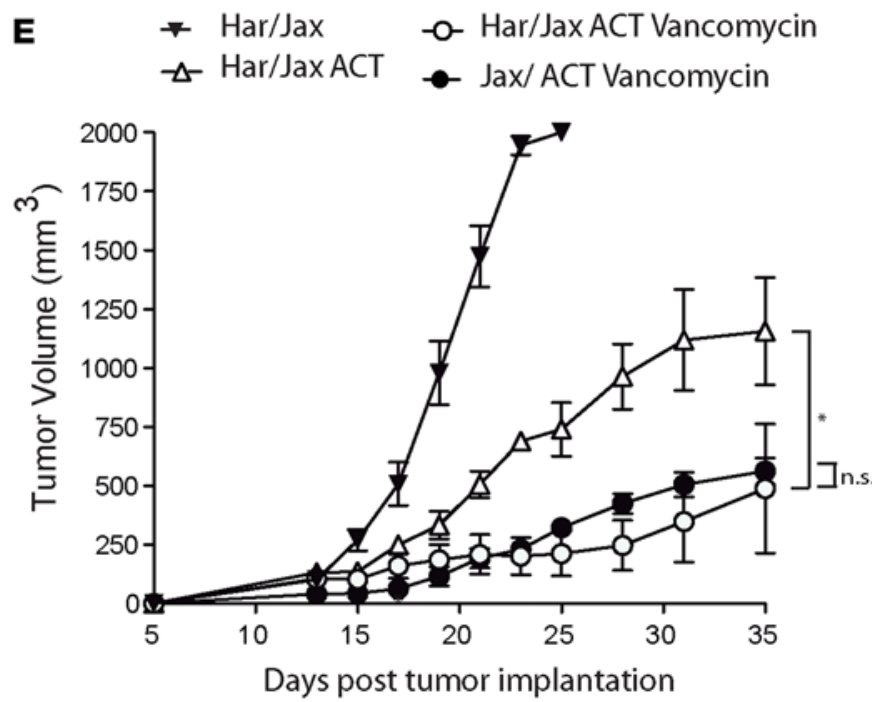

Figure 4. Increased ACT efficacy by antibiotic treatment is phenocopied by fecal microbiota transplant. (A) Detection of bacterial DNA in stool samples by broad-range PCR using universal PCR primers. (B) Detection of segmented filamentous bacteria (SFB) in DNA of stool samples. (C) Principal coordinates analysis ( $P C O A)$ of unweighted UniFrac distance and (D) between-group distances for samples from Jackson donor mice, Harlan recipient mice, and native Harlan mice. Day 0 data is shown in red; Day 7 data is shown in blue. (E) Tumor growth of Harlan mice reconstituted with Jackson microbiota. Tumor growth data are representative of 2 independent experiments with 5 mice per group. Means \pm SEM are shown. Differences in tumor volume were evaluated with linear mixed effects models. ${ }^{*} P<0.05$.

Gut microbiota composition influences tumor infiltration and systemic expansion of reactive $T$ cells. Given the importance of $\mathrm{T}$ cell infiltration into the tumor for the effectiveness of ACT, we investigated both total T cell and anti-HPV E7 tetramer-specific T cell infiltration using IHC and flow cytometry analysis in Jax mice. $\mathrm{CD}^{+} \mathrm{T}$ cell infiltration was augmented in $\mathrm{ACT}$ and vancomycin mice compared with vancomycin $(P=0.01)$ or ACT-only groups $(P=0.03$, Figure $5 \mathrm{~A})$. The specificity of this antitumor infiltration was assessed by staining with the HPV E7-specific tetramer. The number of E7-specific $\mathrm{CD}^{+} \mathrm{CD}^{+} \mathrm{T}$ cells was increased in the tumors of mice treated with the combination of ACT and vancomycin compared with ACT and Neo/Met $(P=0.02)$ or ACT alone $(P=0.02$, Figure $5 \mathrm{~B})$. To understand if this was due to preferential in vitro expansion of CD8 T cells, we performed flow cytometry immune phenotyping after the in vitro polarization/expansion (Supplemental Figure 6). The results show that in vitro polarization/expansion, before adoptive transfer, does not preferentially expand $\mathrm{CD} 8^{+} \mathrm{T}$ cells. Thus, in spite of expansion of both $\mathrm{CD} 4^{+}$and $\mathrm{CD} 8^{+}$subsets, only $\mathrm{CD} 8^{+} \mathrm{T}$ cells specifically accumulate within the tumor. Host myeloid-derived suppressor cells (MDSC), macrophages, and host $\mathrm{CD} 8^{+}$and $\mathrm{CD} 4^{+} \mathrm{T}$ cells were analyzed as well in tumor (Supplemental Figure 7) and spleen (Sup- 
A

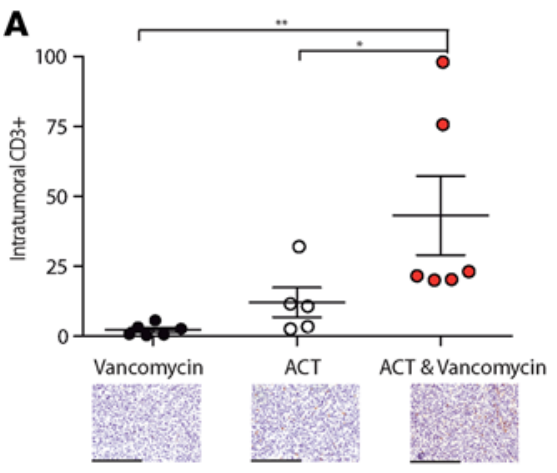

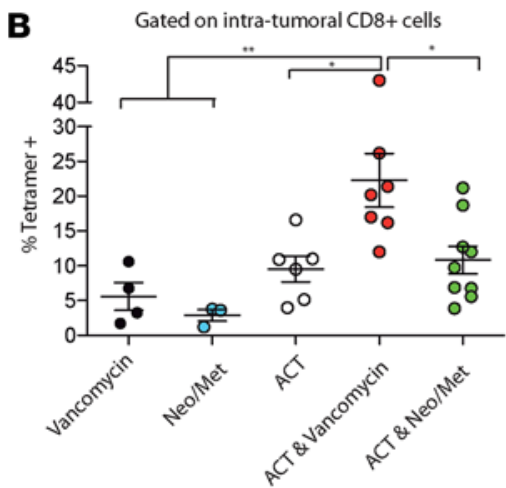
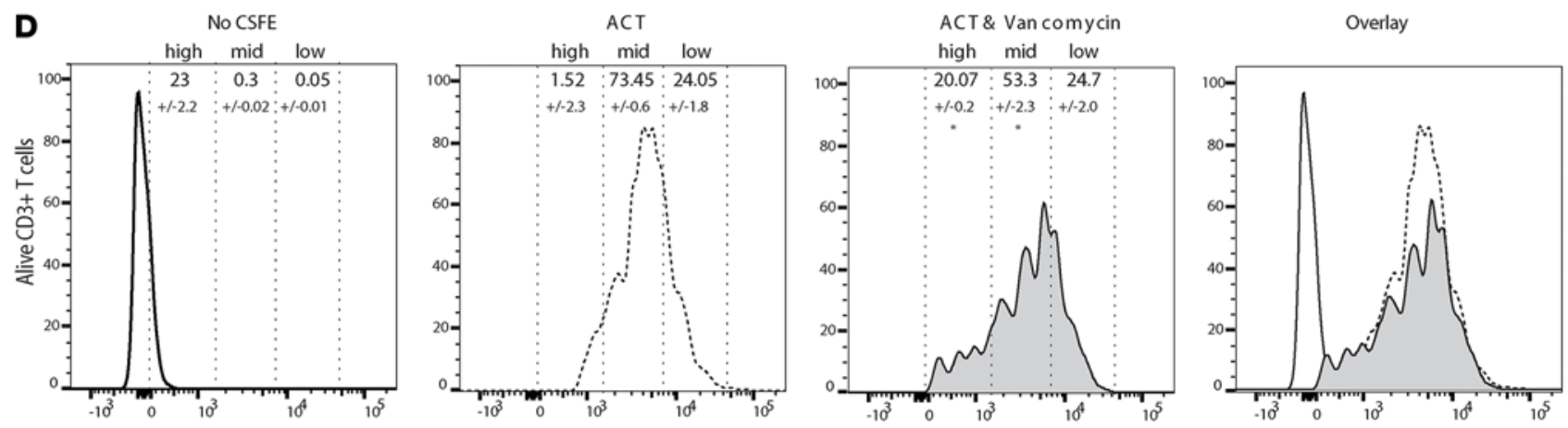

CSFE

Figure 5. Microbiota composition influences both tumor infiltration and systemic expansion of reactive T cells. (A) IHC quantification of $C D 3^{+} T$ cell infiltration within TC1 tumors and representative CD3 staining images. Scale bars: $200 \mu \mathrm{m}$ (original magnification, 20x). Each dot represents a mouse; mean of 5-6 fields per mouse and 5-7 mice per group. Means \pm SEM are shown from 1 representative experiment out of 3. (B) Intratumoral CD8 ${ }^{+}$T cells E7 ${ }_{49-57}$ tetramer-positive and (C) IFN- $\gamma$ ELISPOT of splenocytes from TC1 tumor-bearing mice treated with ACT and vancomycin or Neo/Met. Each dot represents a mouse; 3-9 mice per group. Means \pm SEM are shown from 1 representative experiment out of 3. (D) Proliferation of CFSE-labeled adoptively transferred T cells was measured by flow cytometry based on CFSE dilution peaks 8 days after ACT. Representative histograms. Numbers represent the average (2 independent experiments, $n=3$ ) of percentage on each proliferation group (low, mid, and high). A 2-tailed $t$ test analysis was performed. ${ }^{*} P<0.05$, ${ }^{* *} P<$ $0.01,{ }^{* * *} P<0.001$.

plemental Figure 8), but only $\mathrm{T}$ cells and $\mathrm{CD} 8^{+}$tetramer ${ }^{+} \mathrm{T}$ cells were significantly increased in tumor with the addition of vancomycin to ACT treatment (Figure 5, A and B).

We next examined whether antibiotic treatment influenced the systemic expansion of reactive $\mathrm{T}$ cells. Using an IFN- $\gamma$ ELISPOT assay, we found that splenocytes from TC1 tumor-bearing mice treated with $\mathrm{ACT}$ and vancomycin exhibited an increased response against the $\mathrm{E} 7_{49-57}$ epitope compared with the individual treatments $(P=0.005$ for ACT, $P<0.001$ for vancomycin) or the combination with ACT and Neo/Met $(P<0.001$, Figure $5 \mathrm{C})$. There was no statistically significant difference between mice treated with ACT and those treated with ACT and Neo/Met $(P=0.11)$. We next measured cell division by staining adoptively transferred $\mathrm{T}$ cells with CFSE. T cells were adoptively transferred into TC1 tumor-bearing mice treated with vancomycin and untreated mice. Eight days after ACT, cells were recovered from spleen. T cells that had been adoptively transferred into TC1 tumor-bearing mice treated with vancomycin diluted CSFE more than T cells from untreated TC1 tumor-bearing mice (Figure 5D), confirming - together with the ELISPOT and the tetramer staining - an increased proliferation of the adoptively transferred $\mathrm{T}$ cells. Thus, depletion of vancomycin-sensitive populations from the gut microbiota of Jax mice increased systemic reactive $\mathrm{T}$ cells and therapy effectiveness.

Increased expression of genes associated with cytotoxic and Th1 profiles in TC1 tumors treated with ACT and vancomycin. The efficacy of immunotherapy against established tumors depends on lymphocyte recruitment and expansion, effector function, and local tumor microenvironment remodeling (2). Effector functions include cytotoxicity, mediated by perforin/granzyme pathways, and Th1 cytokines such as IFN- $\gamma$ and IL-12 (45-47). In order to investigate whether gene expression signatures were altered with the addition of vancomycin, we 
A

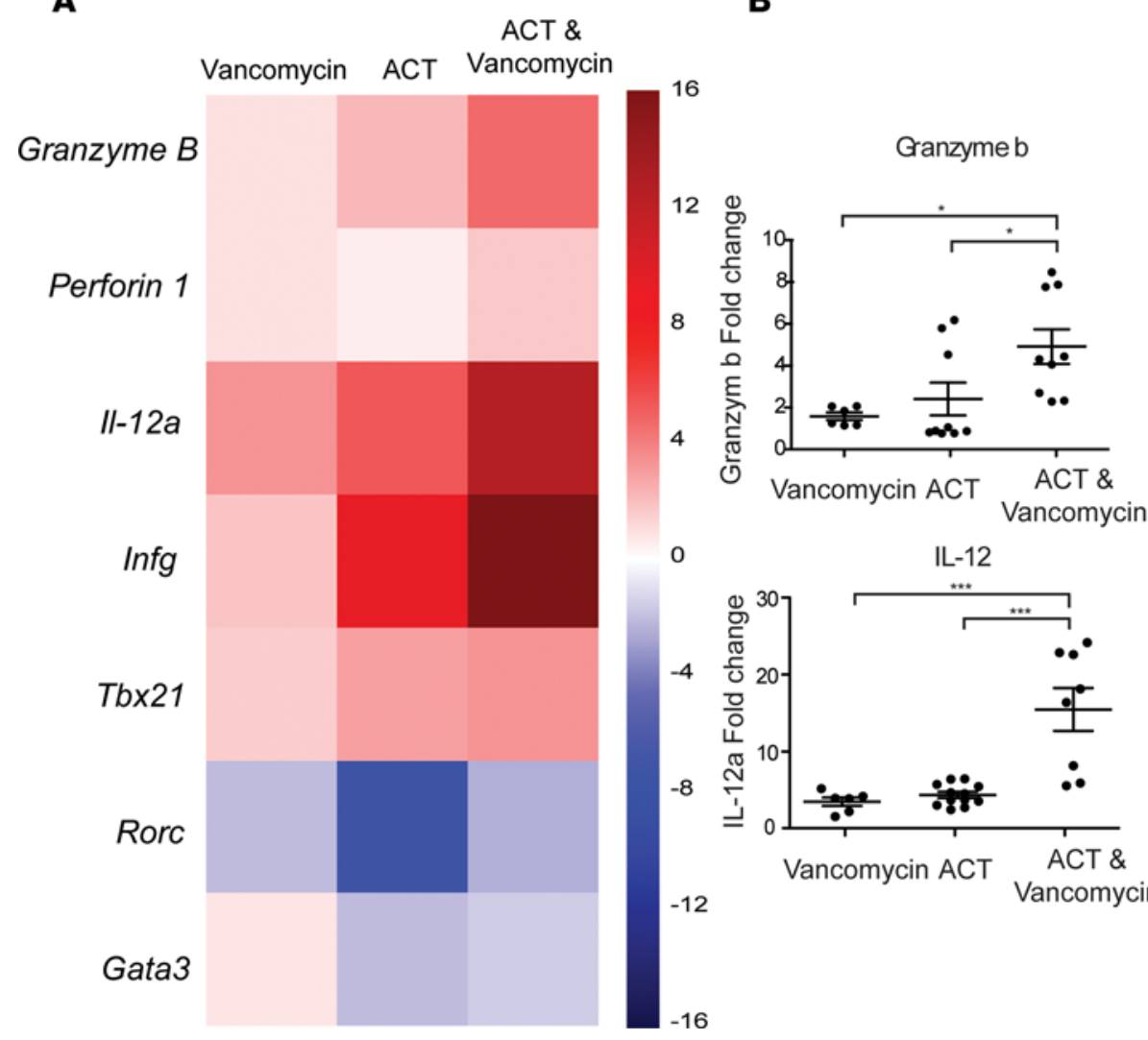

\section{B}


A

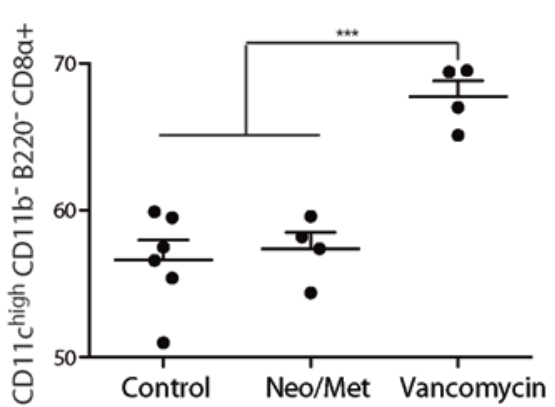

C

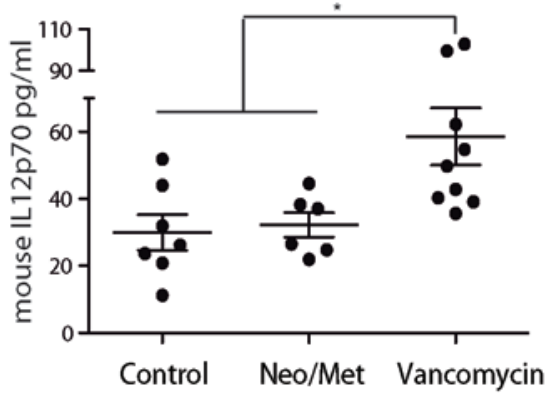

B

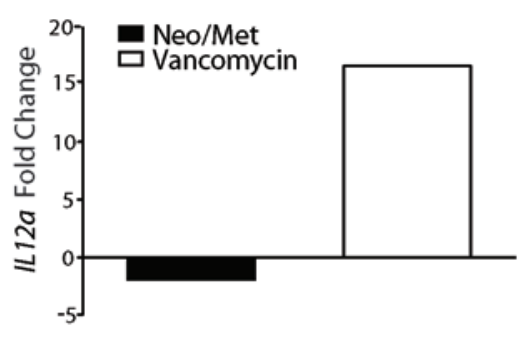

D

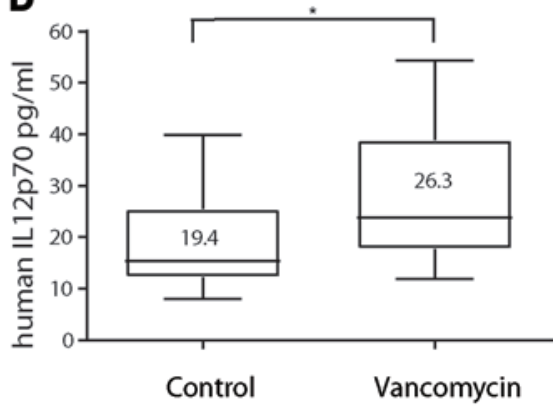

Figure 7. Vancomycin treatment increases the number of systemic CD8 $\alpha^{+}$DC. Jackson mice were treated for 10 days with antibiotics. (A) Phenotype analysis of splenic DC population by flow cytometry, $\mathrm{CD} 8 \alpha^{+} \mathrm{DC}$ subset shown (CD11c ${ }^{\text {high }}$ CD8 ${ }^{+}$B220-CD11b-). (B) I/12a RNA expression of purified CD11 $\mathrm{C}^{+} \mathrm{DC}$ (pooled by group). (C) Mouse IL-12p70 protein serum levels. (D) Human IL-12p70 serum levels on patients undergoing the same therapy, allogeneic hematopoietic cell transplantation, and treated with vancomycin. (E) $\mathrm{E7}_{49-57} \mathrm{IFN}-\gamma$ ELISPOT of purified

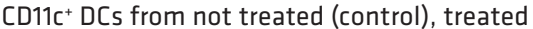
with $\mathrm{Neo} /$ Met or vancomycin ( \pm the addition of $1 \mu \mathrm{g} / \mathrm{ml}$ of blocking monoclonal antibody IL-12p70) mice and incubated with T cells from immunized mice. Mouse data is representative of 3 independent experiments with 4-9 mice per group. Each dot represents a mouse; means \pm SEM are shown. A multiple $t$ test analysis was performed. ${ }^{*} P<0.05,{ }^{* *} P<0.001$

\section{E}

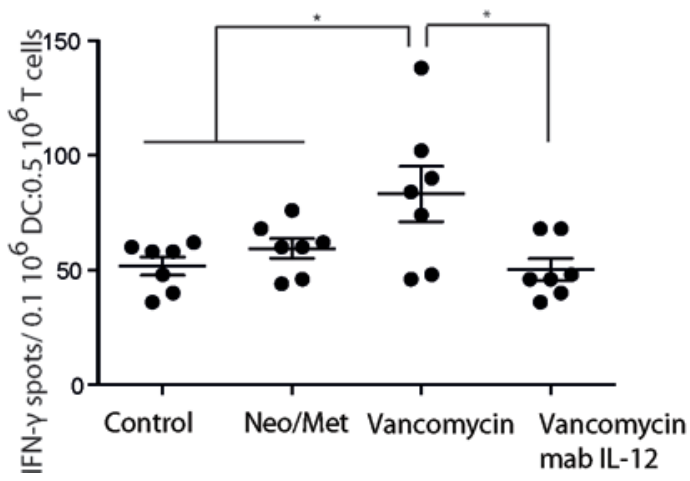

To demonstrate a similar effect of vancomycin on IL-12p70 cytokine levels in humans, we turned to a cohort of patients undergoing allogeneic hematopoietic cell transplantation for hematologic malignancies at University of Pennsylvania (Philadelphia, PA, USA). In December 2015, we instituted a uniform policy of oral vancomycin prophylaxis against Clostridium difficile. This allowed us to compare IL-12p70 levels in patients receiving oral vancomycin with those in historical controls who did not receive oral vancomycin but underwent identical treatment. We observed an increase of IL-12p70 levels in serum when patients were treated with vancomycin $(P=0.03$, Figure $7 \mathrm{D}$ and Supplemental Table 1). This suggested that oral vancomycin administration in humans increased the IL-12 concentration in serum, as we observed in mice.

The gut microbiota can trigger TLR4 signaling and induce IL-12 production systemically after total body irradiation (TBI) $(30,51)$. To investigate the role of TLR4 in microbiota-induced alterations of ACT efficacy, we tested the effects of vancomycin treatment in Tlr4-KO mice. Tlr4-KO mice treated with vancomycin presented increased levels of IL-12p70 protein in serum relative to Tlr4-KO untreated mice (Supplemental Figure 10), following the same pattern of WT mice. Thus, induction of TLR4 was ruled out as the mechanism leading to increased IL-12 levels under vancomycin treatment.

To further investigate the effects of antibiotic treatment on the antigen-presentation function of DCs, we tested the immune response of freshly isolated $\mathrm{T}$ cells from immunized mice against DCs isolated from spleens of mice treated with vancomycin or Neo/Met using IFN- $\gamma$ ELISPOT. Purified T cells had a higher response when the peptide $\mathrm{E} 7_{49-57}$ was presented by DCs derived from vancomycin-treated mice compared with untreated 
A
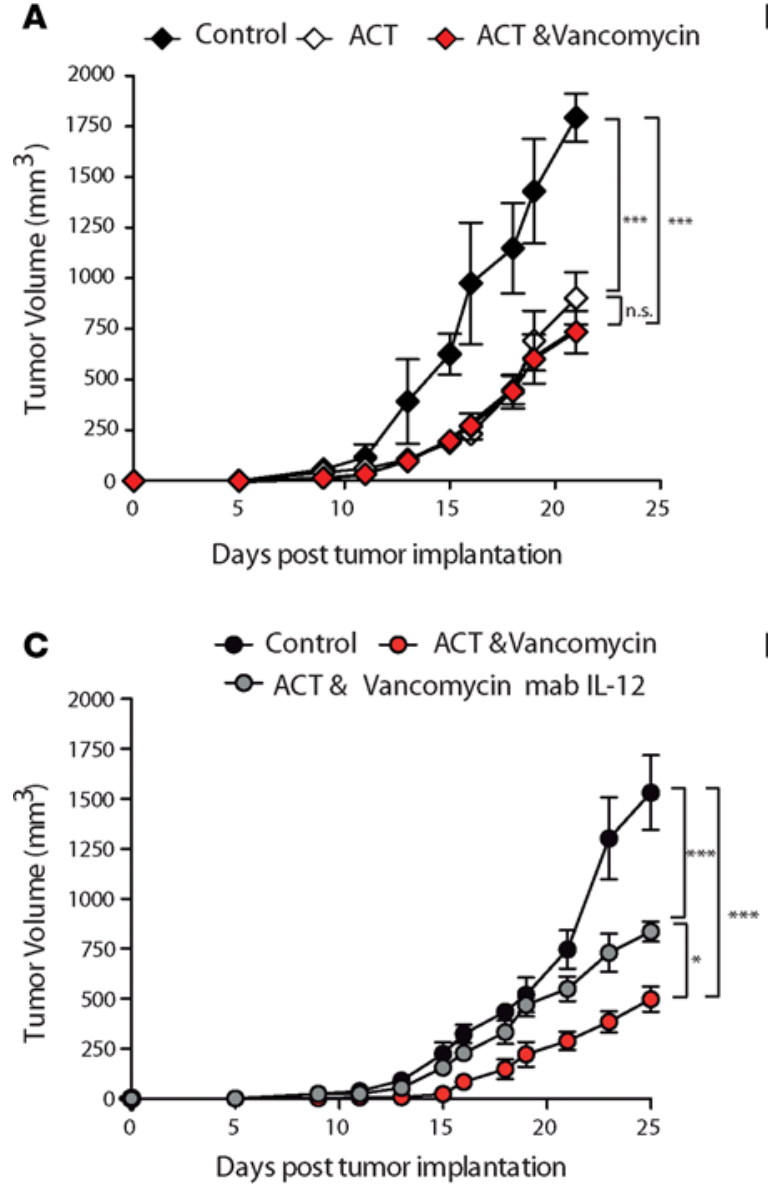

B

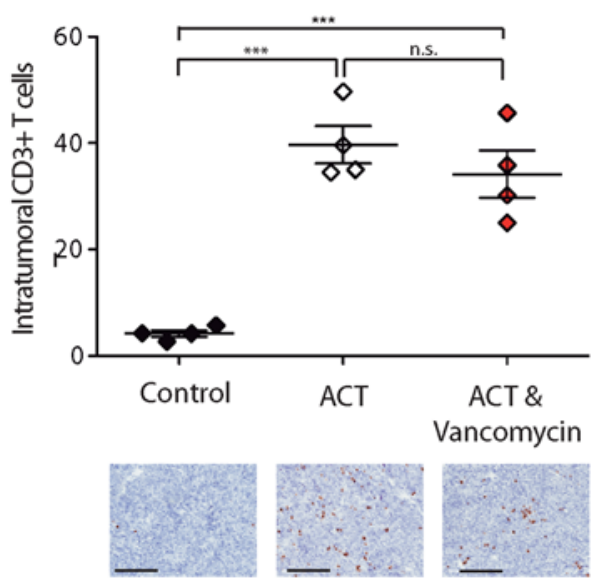

D

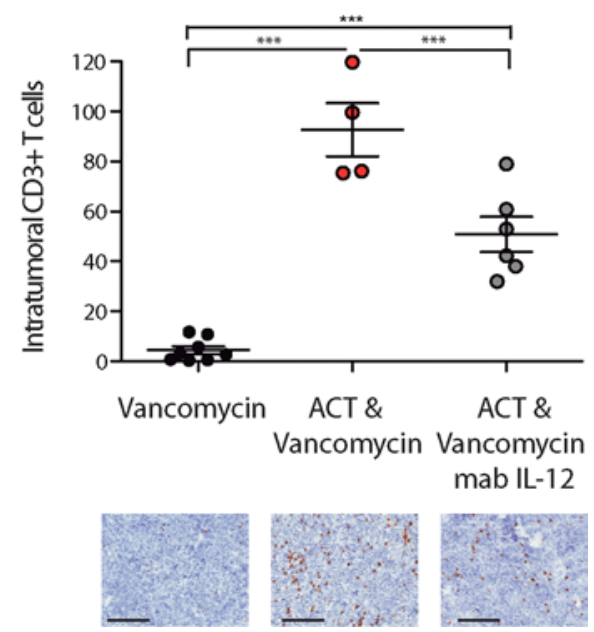

Figure 8. Effects of the microbiota during adoptive T cells transfer therapy depend on IL-12. (A and B) Jackson IL-12-KO and (C and D) C57BL/6 mice treated with a monoclonal IL-12 blocking antibody. ( $\mathbf{A}$ and $\mathbf{C}$ ) tumor growth data are representative of 2 independent experiments with 5 mice per group; means \pm SEM are shown. (B and D) IHC quantification of $\mathrm{CD}^{+}$T cell infiltration within TC1 tumors and representative CD3 staining images. Scale bars: $200 \mu \mathrm{m}$ (original magnification, 20x). Each dot represents a mouse; mean of 5-6 fields per mouse and 4-7 mice per group. Means \pm SEM are shown. Differences in tumor volume were evaluated with linear mixed effects models. For other panels, a multiple $t$ test analysis was performed. ${ }^{*} P<0.05,{ }^{* *} P<0.01,{ }^{* *} P<0.001$.

mice $(P=0.03$, Figure 7E) or mice treated with Neo/Met antibiotic cocktail $(P=0.04)$. The heightened response of $\mathrm{T}$ cells in vancomycin-treated mice was abolished by the addition of IL-12 blocking antibody $(P=0.03$, Figure 7E). Overall, these data suggest that microbiota dysbiosis has a robust impact on the $\mathrm{CD} 8 \alpha^{+} \mathrm{DC}$ subpopulation, which in turn upregulates IL-12p70, leading to an enhanced T cell response.

Effects of gut microbiota on adoptive transfer therapy depends on IL-12. To validate the role of IL-12 on microbiota effects on ACT, we compared TC1 tumor growth in C57BL/6 IL-12-KO mice, which received ACT in combination with vancomycin treatment, with mice receiving no antibiotics. Vancomycin treatment did not improve ACT efficacy when the $I 112$ gene was knocked out, as measured by tumor progression $(P=0.32$, Figure $8 \mathrm{~A}$ ) or T cell infiltration $(P=0.26$, Figure $8 \mathrm{~B})$, but ACT maintained its effects $(P<0.001)$. In order to confirm the importance of this cytokine in the synergy, we injected a blocking antibody for IL-12 into TC1-bearing mice that received ACT and vancomycin. The positive effect of vancomycin treatment upon tumor growth and $\mathrm{T}$ cell infiltration was lost, relative to mice who had not received the blocking antibody ( $P$ $=0.02$, Figure $8 \mathrm{C} ; P<0.001$, Figure $8 \mathrm{D}$ ). Together, these results demonstrate that IL-12 contributes to the microbiota-associated antitumor effect of vancomycin treatment in ACT.

\section{Discussion}

In this study, we describe the impact of the gut microbiota composition on ACT and demonstrate that the microbiota can be altered to improve ACT efficacy. Using the HPV E6/7-expressing cervical cancer 
TC1 model, we report that ACT in tumor-bearing mice is significantly affected by differences in the native composition of the gut microbiota, treatment with antibiotics, and heterologous fecal transfer. ACT efficacy was increased when the bacterial community composition was altered with vancomycin, but it was unchanged when a combination of neomycin and metronidazole was applied. Improvement in ACT efficacy was associated with an increase of systemic CD $8 \alpha^{+}$DCs and IL-12p70 levels, and with more effective expansion of adoptively transferred antitumor $\mathrm{T}$ cells in the animals receiving vancomycin.

Several recent studies have demonstrated the impact of the gut microbiome on cancer immunotherapies (52). In 2013, Iida et al. reported that the gut microbiota played a role in activating the innate immune response against cancer (29). Following s.c. injection of tumor cells in mice, they observed that CpG-oligonucleotide immunotherapy in combination with platinum chemotherapy had a reduced efficacy after antibiotic treatment with a cocktail of vancomycin, neomycin, and imipenem. Control mice in their study harbored a range of Bacteroidetes and Clostridiales species, similar to Har mice in our experiments, which responded optimally to ACT. They reported that individual antibiotics increased the variability of TNF response but did not measure tumor growth in their model after exposure to individual antibiotics. In contrast to their study, we found that the response to ACT could be modulated by specific antibiotic treatments. In a different mouse model, Viaud et al. reported associations between the small intestine microbiota and the efficacy of antitumor therapy with cyclophosphamide (CTX) (53). At the level of the gut microbiome composition, they observed modest differences in small intestinal microbiota and no difference in cecal microbiota composition, while we found that the fecal microbiota changed drastically in our context. In an approach similar to ours, Viaud et al. applied several antibiotic regimens to alter the gut microbial composition of tumor-bearing mice. Application of vancomycin abrogated the response to CTX therapy. In their model, the response to CTX therapy was mediated by different bacteria and host immune cell populations than the response to ACT in our experiments. Importantly, they demonstrated that only a specific phenotype of T cells, pathogenic and not naive Th17 T cells, could restore the CTX efficacy when administrated with vancomycin. In our experiment, we utilized both $\mathrm{CD}^{+}$nonpolarized and $\mathrm{T}$ cells polarized under Th1 conditions in vitro and detected no Th17 signature in treated tumors. Thus, these differences could account for the different outcome when applying vancomycin.

The effect of specific Bacteroides species on tumor growth in mice treated by CTLA-4 blockade was reported by Vétizou et al. (27). Elimination of the species by broad-spectrum antibiotics eliminated the response to therapy, while introduction of $B$. fragilis bacteria or associated molecules restored the response phenotype. In our experiments, we observed a range of Bacteroides species in Har mice but not in Jax mice, correlating with response to ACT. However, we observed that the Bacteroides genus was eliminated after treatment with vancomycin, ruling out species like $B$. fragilis as a mechanism for the observed vancomycin-induced response to ACT. In this study, a number of related species in the uncultured Bacteroidales S24-7 family were found to be associated with reduced tumor growth in the vancomycin-treated groups, representing an area of potential overlap with the results in our study.

The genus Bifidobacterium was a particular point of interest for Sivan et al., who carried out a study on PD-L1 blocking antibody therapy in a mouse model of melanoma (28). In their experiments, Jax mice responded more favorably to therapy than mice purchased from Taconic. In our study of response to ACT, Jax mice performed less favorably than Har mice. Furthermore, the genus Bifidobacterium was not prominent in the 16S rRNA marker gene sequencing results from either Jax or Har mice in our experiments; thus, it was ruled out as a potential explanation for the differential response to ACT. However, both studies underline the critical role of the gut microbiota on DCs in the modulation of the antitumor immune response. In addition to providing direct evidence for the impact of the gut microbiota on antitumor immune response, several studies have demonstrated the relevance of gut bacteria to immune development and homeostasis and to $\mathrm{T}$ cell differentiation and function $(22,25,26,54-56)$.

To date, numerous HPV E7 DNA-based vaccine design modifications and approaches (i.e., codon optimization, antigen targeting modification, or coinjection of adjuvants) have been shown to augment HPV antigen-specific CTL responses (57-59). These studies show that antigen-specific immune responses in humans and mice generated by HPV E7 vaccines increases tumor cure rates and long-term memory. In the TC1 model, the elicitation and tumor infiltration by antigen-specific CD8 $8^{+} \mathrm{T}$ cells is essential for the observed antitumor effects of the HPV E7 vaccine. In alignment with the TC1 model, our results show a specific increase of $\mathrm{CD}^{+} \mathrm{T}$ cells within the tumor. Given that the efficacy of $\mathrm{T}$ cell immunotherapy against established tumors depends on Th1 polarization and cytotoxicity, via the perforin/granzyme system (60- 
63), our results make a significant contribution to the field as we confirm that dysbiosis caused by vancomycin treatment is associated with increased expression of the cytolytic/Th1 signature in ACT-treated tumors outside the gastrointestinal tract. Although significant effects were not observed in the control group, only TBI mice in this context, we cannot ignore the possibility that vancomycin treatment also affects the function or numbers of residual endogenous T cells and contributes to this phenotype, as well.

DCs are central in $\mathrm{T}$ cell activation and functions, and they are also influenced by the host gut microbiota (64). We found an increase in the CD $8 \alpha^{+}$DC population in splenic DCs from vancomycin-treated mice. These results align with previous studies, which - although in a different context - found that DCs mediated the effects of the microbiota on tumor development enhancing specific effector T cells $(28,53)$. We also showed that purified $\mathrm{T}$ cells have a greater response when the HPV peptide is presented by DCs derived from vancomycin-treated mice, and the enhanced response was abolished by the addition of IL-12 blocking antibody.

IL-12 is mainly produced by monocyte populations and DCs in response to bacterial products or intracellular pathogens, or upon interaction with activated T cells. Moreover, it is essential for the differentiation, proliferation, and maintenance of Th1 responses that lead to IFN- $\gamma$ and IL-2 production $(65,66)$. In our study, the increase in $I l 12 a$ transcripts by DCs isolated from vancomycin-treated mice was associated with an increase in IL-12p70 protein in serum. We confirmed these results comparing the IL-12p70 levels in serum of human subjects undergoing the same therapy, allogeneic hematopoietic cell transplantation for the treatment of hematologic malignancies, who received oral vancomycin for prophylaxis against the common nosocomial pathogen $C$. difficile. These data, although in a different setting, are encouraging for future studies, as elevated levels of IL-12 in serum are associated with increased numbers of tumor-infiltrating $\mathrm{CD}^{+} \mathrm{T}$ cells and $\mathrm{CD}^{+} \mathrm{T}$ cells in colorectal cancer (67). Additionally, administration of vancomycin was also associated with an increase of host $\mathrm{CD}^{+}$cells in tumor, probably due to a more conductive microenvironment in tumors of mice treated with ACT and vancomycin

Moreover, antibiotic treatment did not improve ACT efficacy when either Il-12 gene expression was knocked out or when IL-12/IL12 receptor was antagonized by blocking antibody administration. Although the IL-12p40 subunit is shared between IL-12 and IL-23 cytokines, their production and secretion in response to cancer is regulated differentially. Additionally, their functions are different and even opposite of one another, as IL-23 is more relevant in the generation of the Th17 response and IL-17 production (68, 69). In our analysis, we could not detect amplification of $I L-17 a$ transcripts in tumors treated with ACT and vancomycin. Taken together, these results demonstrate that IL-12 contributes to the synergistic modulatory effects of vancomycin on ACT therapy and tumor T cell infiltration.

A study performed in B16 tumor-bearing mice identified TLR4 as one possible mechanism through which microbiota could enhance the positive effect of TBI upon tumor progression (30). Within 24 hours from TBI, gastrointestinal epithelia damage induced by radiation causes the gut microbiota to leak into the i.p. space, with consequent transient infection, induction of TLR4, and increase of systemic IL-12 (30). Based on this work, more recently Bowers et al. showed that TBI-induced IL-12 augments Tc17 cell-mediated tumor immunity (51). By utilizing Tlr4-KO animals, our results preclude this signaling as a mechanism responsible for the increased levels of IL-12p70 in vancomycin-treated mice. However, our data do not exclude other TLRs, NLRs or other damage-associated molecular patterns (DAMPs), as bacteria have a number of ligands beyond LPS that can be responsible for the observed effects.

Evidently, the response to antitumor therapies in mice is mediated by a complex range of microbes and immune system interactions. As our study highlights, outcomes in mice are influenced by the tumor model, the therapeutic design, and the gut microbial composition. Intervention with targeted antibiotic therapies has produced consistent results within each context and therefore will remain an important method for altering the composition of complex microbial communities in the gut until specific sets of bacterial species can be identified and cultured in standard collections The lack of available strains for Bacteoidales S24-7, a prominent family in the previous studies cited here and a candidate for differential response to ACT in our study, highlights the difficulty in moving forward with cultured organisms alone.

A better understanding of the mechanisms by which the gut microbiome regulates the efficacy of ACT will help to maximize the effectiveness of immunotherapies in the future (70). Moreover, we propose that the gut microbiome will need to be modulated in a therapy-specific manner. The present study underscores the importance of the gut bacteria to ACT immunomodulation via IL-12-mediated effects on systemic expansion and tumor invasion of the adoptively transferred $\mathrm{T}$ cells. 


\section{Methods}

Mouse strains and cell lines. Six- to 8-week-old C57BL/6 (H-2 ${ }^{\mathrm{b}}$ ), IL-12p40-KO (strain B6.129S1-I112btm$1 \mathrm{Jm} / \mathrm{J}$ ), and TLR4-KO (strain B6B10ScN-Tlr4<lps-del $>/ \mathrm{JthJ}$ ) female mice were purchased from Jax and Har. Har mice were sourced from the company's Indianapolis (Indiana, USA) facility and were DOCK2 WT. Mice were randomly assigned to different experimental groups before tumor challenge. No animals were excluded from the analysis. Mice from different vendors were kept separately, and procedures were established to avoid microbiota transfer among them; only 1 cage was inside the hood at a time, gloves were removed between cages, and chlorine dioxide-based sterilant (clydoxS) was used for hood and cages decontamination. Autoclavable chews were utilized instead of nestles, and individual autoclaved food and water bottles (not automated watering system with drinking valves) were employed for the experiments. The TC1 cells (ATCC), a cervix and lung tumor cell line transformed by HPV E6 and E7 viral oncogenes, were propagated in $5 \% \mathrm{CO}_{2}$ at $37^{\circ} \mathrm{C}$ and cultured in RPMI 1640 medium with $2 \mathrm{mM}$ L-glutamine (Corning) supplemented with 10\% FBS (GIBCO, 1456821), adjusted to contain $1.1 \mathrm{~g} / 1$ sodium pyruvate and $10 \mathrm{mM}$ HEPES (Corning), and supplemented with $2 \mathrm{mM}$ nonessential amino acids (GIBCO) and $100 \mathrm{U} / \mathrm{ml}^{-1}$ penicillin and $100 \mathrm{mg} / \mathrm{ml}^{-1}$ streptomycin (Corning). TC1 cells were tested to discard mycoplasma contamination.

Immunization. Six- to 8-week-old C57BL/6 mice (Jax) were immunized 3 times (once a week) with 100 $\mu \mathrm{g}$ control plasmid (pCon) E6-E7 DNA plasmid (39) (encoding for E6 and E7 genes) i.m. and sacrificed 1 week after the last immunization.

Tumor challenge. Six- to 8-week-old C57BL/6 mice (Jax and Har) were irradiated with a total dose of $4 \mathrm{~Gy}$ and, 48 hours later, were injected s.c. with $1 \times 10^{5} \mathrm{TC} 1$ cells per mouse. Solid tumor development was monitored by caliper measurements every other day from the day they were palpable. Experiments were terminated when control mice tumors reached $1.75-2 \mathrm{~cm}^{3}$ (according to University of Pennsylvania guidelines).

Antibiotic administration. Mice received drinking water containing vancomycin $(0.5 \mathrm{~g} / 1)$, neomycin $1 \mathrm{~g} / 1$ plus metradonizole $1 \mathrm{~g} / 1$ (MilliporeSigma), or water alone ad libitum for 10 days. The Neo/Met treatment water was supplemented with $1.5 \mathrm{~g} / 1$ of dextrose, maltodextrin, and sucralose mix.

Adoptive T cell therapy. Adoptive T cells transfer was performed 5 days after tumor implantation. Single cell suspension of spleen cells from immunized C57BL/6 Jax mice $(n=5)$ were pooled and used to magnetically isolate the lymphocyte population (CD3 ${ }^{+} \mathrm{T}$ cells) (MiltenyBiotec, catalog 130-095-130). Cells (2.5 $\times 10^{6} \mathrm{cells} / \mathrm{ml}$ ) were seeded into 24 -well plate, previously coated with mouse anti-CD3 antibody (clone 17A2, eBioscience), with Th1 polarizing media, composed by rmIL-12 (3.3 ng/ml; Peprotech, catalog 21012) and mouse anti-IL-4 (10 $\mu \mathrm{g} / \mathrm{ml}$; clone 11B11, BioXCell). On the second day of culture, T cells were split and rmIL-2 (0.6 ng/ml; Peprotech, catalog 212-12) was added to the polarizing media. After 3 days of polarization in vitro, $5 \times 10^{6} \mathrm{~T}$ cells were adoptively transferred via i.v. For ACT with nonpolarized T cells, $5 \times 10^{6}$ of freshly isolated $\mathrm{CD}^{+} \mathrm{T}$ cells were adoptively transferred i.v.

Microbial transfer. Antibiotic-treated animals ( $1 \mathrm{~g} / 1$ each neomycin sulfate and metronidazole, and 0.5 $\mathrm{g} / 1$ of vancomycin hydrochloride [MilliporeSigma] were used for 14 days and provided ad libitum) were gavaged with $200 \mu \mathrm{l}$ of fecal bacteria obtained by homogenizing the full contents of 2 feces pellets from the corresponding donor mice in PBS. Recipient mice were housed in a single cage. Fecal samples were collected from donor and recipient mice, allowing the fecal donor mice to serve as a control group for their respective vendor. Detection of universal bacteria (forward, 5'-AGGAGGTGATCCAACCGCA-3'; reverse, 5'-AACTGGAGGAAGGTCGGGAY-3') (71) and Segmented Filamentous Bacteria (forward, 5'-GACGCTGAGGCATGAGAGCAT-3'; reverse 5'-GACGGCACGGATTGTTATTCA-3') (72) was performed as previously described. Briefly, DNA was isolated from mouse stools at different time points using the QIAamp DNA stool extraction kit (Qiagen, catalog 51504), according to manufacturer's instructions, and standard PCR was performed. PCR products were analyzed by agarose gel electrophoresis and ethidium bromide (EB) staining.

In vivo IL-12 blocking treatment. Mice received via i.p. injections 3 doses (once a week) of $1 \mathrm{mg}$ of monoclonal blocking antibody (InVivoMAb anti-mIL-12p75, clone R2-9A5, BioXCell).

ELISpot. Ninety-six-well MAIP plates (MilliporeSigma, catalog MAIPS 4510) were coated overnight with a $2.5 \mu \mathrm{g} / \mathrm{ml}$ solution of rat anti-mouse IFN- $\gamma$ (IgG1, clone R4-6A2, BD Biosciences). Bulk splenocytes were plated at $0.5 \times 10^{6}$ cells/well in triplicate and incubated for 20 hours at $37^{\circ} \mathrm{C}$ with $2 \mu \mathrm{g} / \mathrm{ml}$ peptide (E7 peptide [RAHYNIVTF]) synthesized by JPT (https://www.jpt.com). For the DC assay, DCs were isolated from splenocytes using mouse CD11c MicroBeads (Milteny Biotec, catalog 130-097-059) 
according to manufacturer's instructions. DCs $\left(0.1 \times 10^{6}\right)$ were plated in triplicate and incubated 20 hours at $37^{\circ} \mathrm{C}$ with $0.5 \times 10^{6} \mathrm{CD}^{+} \mathrm{T}$ cells from immunized mice and $2 \mu \mathrm{g} / \mathrm{ml}$ of E7 peptides. To block IL-12 in vitro, $10 \mu \mathrm{g} / \mathrm{ml}$ monoclonal antibody IL-12p75 was added to each well (InVivoMAb anti-mIL-12p75, clone R2-9A5, BioXCell). After incubation, plates were washed with PBS and 0.05\% Tween 20 (Bio-Rad) and incubated 3 hours at room temperature with anti-mouse biotin-conjugated anti-IFN- $\gamma$ antibody (rat IgG1, clone XMG1.2, BD Biosciences); Streptavidin-alkaline phosphatase conjugate (BD Biosciences, catalog 554065) was added for 30 minutes. Plates were developed by adding nitroblue tetrazolium/5-bromo-4-chloro-3-indolyl phosphate (Thermo Fisher Scientific, catalog 34042), and spots were then counted using an automated ELISpot reader (AutoimmunDiagnostika $\mathrm{GmbH}$ ).

Immunohistochemical tumor analysis. Tumors were embedded in OCT medium and immediately snap frozen in dry ice. Sections (6- $\mu \mathrm{m}$ thick) were stained for mouse CD3 (CD3 [Early T cell Marker], Rabbit Monoclonal Antibody, RM-9107-S0, Thermo Fisher Scientific) with hematoxylin as a counterstain. Images of the slides were taken using a Nikon Eclipse TI microscope. Investigators performed blind assessment of IHC sections.

Flow cytometry. Cells were subjected to up to 7-parameters flow cytometry on a FACSCanto flow cytometer using BD FACSDiva software (BD Biosciences); data were analyzed using FlowJo version X (Tree Star Inc.). The following monoclonal antibodies against mouse markers were used: PercP/Cy 5.5-conjugated anti-CD8 (clone 53-6.7, BD Pharmingen), APC-conjugated anti-CD3ع (clone 145-2C11, BD Pharmingen), FITC-conjugated anti-CD4 (clone RM4-5, BioLegend), and Brilliant Violet-510-conjugated CD45 (clone 104, BioLegend). iTAg Tetramer/PE - H-2 Db HPV 16 E7 (RAHYNIVTF) (MBL) was used to detect specifically infiltrating E7 CD3 T cells adoptively transferred to mice.

LIVE/DEAD Fixable Aqua Dead Cell Stain (Invitrogen) was used to gate alive cells, and CellTrace CFSE was used to track adoptively transferred cells and proliferation (Invitrogen). DC phenotyping was done using the following monoclonal antibodies against mouse markers: PE-Cy7-conjugated CD11c (clone N418 eBioscience), APC-conjugated CD11b (M1-70, BioLegend), PercP/Cy 5.5-conjugated antiCD8 (clone 53-6.7, BD Pharmingen), APC Cy7-conjugated B220 (clone RA36B2, BioLegend), FITC-conjugated GR-1 (clone RB6-8C5, BioLegend), PE-conjugated F4/80 (clone BM8, BioLegend).

Gating strategy. Lymphocytes were determined by the forward and side scatter profile. T cells were assessed after gating for live cells (and $\mathrm{CD} 45^{+}$cells in tumor) with anti-CD3 $\varepsilon$ and anti-CD8. Percentage of tetramer-HPV-specific T cells was quantified inside CD3ع/CD8 $\alpha$ cell subset. DC subset was determined by anti-CD11c and anti-CD11b inside live cells subset. CD $8 \alpha^{+}$DCs were assessed by anti$\mathrm{CD}^{+}$staining inside $\mathrm{CD} 11 \mathrm{c}^{+} \mathrm{Cd} 11 \mathrm{~b}^{-} \mathrm{B} 220^{-}$compartment.

Mouse IL-12 ELISA. Mouse IL-12 was measured from serum using a DuoSet ELISA mouse IL-12p70 kit according to the manufacturer's instruction (R\&D Systems, catalog DY419-05).

Human IL-12 ELISA. Human IL-12 levels were measured from plasma using a Human IL-12 p70 Quantikine HS ELISA Kit according to the manufacturer's instruction (R\&D Systems, catalog HS120).

Vancomycin treatment of human patients. From December 29, 2015, all patients undergoing allogeneic hematopoietic cell transplantation received oral vancomycin suspension of $125 \mathrm{mg}$ twice daily beginning on the day of admission for transplantation and continuing until discharge. Vancomycin administration was performed as part of a unit-wide policy instituted in response to the high incidence $(20 \%)$ of $C$. difficile infection occurring during the inpatient stay. Samples from 17 consecutive patients who received vancomycin prophylaxis and had available day-28 plasma aliquots were used. Samples from 20 consecutive patients who did not receive vancomycin prophylaxis (i.e., underwent allogeneic transplantation prior to the institution of our vancomycin prophylaxis policy) were used. All identifiers from human samples were removed before analysis

Mouse IL-12 gene expression on DCs. Mouse Il12a gene expression on DCs was quantified using $\mathrm{RT}^{2}$ Profiler PCR Array according to manufacturer's instructions (Mouse Cancer Inflammation \& Immunity Crosstalk, Qiagen, catalog 330231 PAMM-181Z).

Molecular signature. The relative quantification of the expression levels of selected genes was carried out by qPCR relative to untreated TC1 tumor controls at day 25 agter tumor implantation, using an ABI PRISM Viia7 (Applied Biosystems). Total RNA from TC1 tumors was extracted using Trizol reagent (Invitrogen) according to the manufacturer's instructions. The purity of the RNA samples was determined using EB visualization of intact $18 \mathrm{~S}$ and $28 \mathrm{~S}$ RNA bands after agarose gel electrophoresis. RNA ( $2 \mu \mathrm{g}$ of total) was used for cDNA synthesis with random primers, using high-capacity cDNA reverse transcription kit (Applied Biosystem, catalog 4368814). cDNA (50 ng) was used in each real-time PCR reaction run according to 
manufacturer's instructions. TaqMan gene expression assays used to quantify expression levels of; Il12a (Mm00434169_m1), Granzyme B (Mm00442834_m1), Tbx21 (Mm00450960_m1), Il4 (Mm00445259_m1), Perforin 1(Mm00812512_m1), Gata3 (Mm00484683_m1), Infg (Mm01168134_m1), IL17a (Mm00439618_ m1), Rorc (Mm01261022_m1), Actin B (Mm00607939_s1), and Gapdh (Mm99999915_g1).

Bacterial $16 S \mathrm{r} R$ A A gene sequencing. Fecal samples were collected from mice housed in a single cage per study group. DNA was extracted from mice samples with the DNA Stool kit (Qiagen) according to the manufacturer's instruction. For $16 \mathrm{~S}$ sequencing analysis, the PCR amplicon was prepared using V4 (5'-TGYCAGCMGCCGCGGTA-3') and V6 (primer 5'-TCACGRCACGAGCTGACG-3') primers with barcodes. The PCR reaction was done using Phusion High-Fidelity DNA Polymerase (New England Biolabs). The PCR reaction was done with an initial step at $98^{\circ} \mathrm{C}$ for 30 seconds, followed by 25 cycles of 10 seconds at $98^{\circ} \mathrm{C}, 20$ seconds at $72^{\circ} \mathrm{C}$, and 5 minutes at $72^{\circ} \mathrm{C}$. The PCR product was purified from excess of primers and DNA contaminants using AMPure XP (Beckman Coulter) according to the manufacturer's instruction; all samples were pooled in equimolar concentrations. The metagenomic sequence reads were generated on the Roche 454 GS FLX.

Sequence reads were analyzed using the QIIME pipeline version 1.8 (73). Reads were discarded if they did not match the expected DNA barcode and primer sequences, contained more than 2 ambiguous base calls (N [IUPAC symbol for an ambiguous base call]), or if the length of $16 \mathrm{~S}$ gene sequence was less than $200 \mathrm{bp}$. Operational taxonomic units were selected by UCLUST (74), using the default parameters in QIIME. Taxonomic assignments were determined by searching the Greengenes 16S database (75), after which taxa were assigned if 2 of the top 3 hits were in agreement (the default method in QIIME). Representative sequences from each operational taxonomic unit (OTU) were aligned with PyNAST (76), and a phylogenetic tree was inferred using FastTree (77). The resultant tree was used to compute unweighted UniFrac distances between each pair of samples (78).

Statistics. Sample sizes were chosen on the basis of pilot experiments and our experience with similar experiments. For tumor progression experiments, we employed linear mixed effects models to evaluate the effect of treatment on tumor size. A likelihood ratio test was used to compare models with and without the treatment term. For other comparisons apart from microbiome sequencing, we used one-way ANOVA to test for an overall difference between groups. If a difference was found in ANOVA, we followed with 2-tailed Student's $t$ tests using a pooled estimate of the variance. Where multiple comparisons were carried out, we adjusted $P$ values using the Holm-Bonferroni method, which controls for the familywise error rate. All $t$ test $P$ values presented are 2 -tailed, ${ }^{*} P<0.05,{ }^{* *} P<0.01,{ }^{* * *} P<0.001$. For $16 \mathrm{~S}$ sequence data, the PERMANOVA test was used to test a null hypothesis of no difference between group centroids based on unweighted UniFrac distance (79). PERMANOVA $P$ values were calculated using 999 random label permutations. To assess the effect of time (a within-subjects variable), time point labels were permuted only within mice.

Study approval. All animal studies were approved by the IACUC and University Laboratory Animal Resources at the University of Pennsylvania. Mice were treated in accordance with University of Pennsylvania guidelines. Patients were collected under a research protocol that was approved by the University of Pennsylvania IRB.

\section{Author contributions}

MUH designed and performed the experiments, analyzed the data, and drafted the manuscript. KB performed microbiota bioinformatics analyses and gave suggestions for the manuscript. SR assisted with the microbiota experiments and gave suggestions for the manuscript. SG assisted with the ACT experiments. SP assisted with the DC experiments. CT performed microbiota bioinformatics analyses. AG and SG provided human patient samples. MAM and JLT gave suggestions for the manuscript and financial support. FDB gave suggestions for the manuscript and helped with critical advice on how to correctly perform the microbiome studies. CHR gave suggestions for the manuscript, with critical advice on $\mathrm{T}$ polarization protocols and $\mathrm{T}$ cell therapy. AF conceived the project, designed the study, supervised project planning and execution, and wrote the manuscript.

\section{Acknowledgments}

We would like to thanks Richard Gallagher and John Facciponte for his help in the final editing of the paper and Ronny Drapkin for his helpful suggestions. This work was supported by Be the Difference Foundation, Teal Tea Foundation, Pennsylvania Department of Health (PA-DOH), and the University of Pennsylvania research fund. 
Address correspondence to: Facciabene Andrea, University of Pennsylvania, Radiation Oncology, Ovarian Cancer Research Center, Smilow CTR, Room 8-133, 3400 Civic Center Boulevard, Philadelphia, Pennsylvania 19104, USA. Phone: 215.746.7071; Email: facciabe@pennmedicine.upenn.edu.

1. Zhang L, et al. Intratumoral T cells, recurrence, and survival in epithelial ovarian cancer. N Engl J Med. 2003;348(3):203-213.

2. Galon J, et al. Type, density, and location of immune cells within human colorectal tumors predict clinical outcome. Science. 2006;313(5795):1960-1964.

3. Dudley ME, et al. Randomized selection design trial evaluating CD8+-enriched versus unselected tumor-infiltrating lymphocytes for adoptive cell therapy for patients with melanoma. J Clin Oncol. 2013;31(17):2152-2159.

4. Dudley ME. Adoptive cell therapy for patients with melanoma. J Cancer. 2011;2:360-362.

5. Rosenberg SA, Dudley ME. Adoptive cell therapy for the treatment of patients with metastatic melanoma. Curr Opin Immunol. 2009;21(2):233-240.

6. Dudley ME, et al. Cancer regression and autoimmunity in patients after clonal repopulation with antitumor lymphocytes. Science. 2002;298(5594):850-854.

7. Dudley ME, et al. Adoptive cell transfer therapy following non-myeloablative but lymphodepleting chemotherapy for the treatment of patients with refractory metastatic melanoma. J Clin Oncol. 2005;23(10):2346-2357.

8. Porter DL, Levine BL, Kalos M, Bagg A, June CH. Chimeric antigen receptor-modified T cells in chronic lymphoid leukemia. NEngl J Med. 2011;365(8):725-733.

9. Maude SL, et al. Chimeric antigen receptor T cells for sustained remissions in leukemia. NEngl J Med. 2014;371(16):1507-1517.

10. Vaccine Therapy in Treating Patients With Stage IV Breast Cancer. ClinicalTrials.gov. https://clinicaltrials.gov/ct2/show/ NCT00791037. Published November 14, 2008. Updated May 25, 2017. Accessed January 30, 2018.

11. Stevanović S, et al. Complete regression of metastatic cervical cancer after treatment with human papillomavirus-targeted tumor-infiltrating T cells. J Clin Oncol. 2015;33(14):1543-1550.

12. Ramos CA, et al. Human papillomavirus type $16 \mathrm{E} 6 / \mathrm{E} 7$-specific cytotoxic T lymphocytes for adoptive immunotherapy of HPV-associated malignancies. J Immunother. 2013;36(1):66-76.

13. Powell DJ, Dudley ME, Hogan KA, Wunderlich JR, Rosenberg SA. Adoptive transfer of vaccine-induced peripheral blood mononuclear cells to patients with metastatic melanoma following lymphodepletion. J Immunol. 2006;177(9):6527-6539.

14. Romano E, et al. MART-1 peptide vaccination plus IMP321 (LAG-3Ig fusion protein) in patients receiving autologous PBMCs after lymphodepletion: results of a Phase I trial. J Transl Med. 2014;12:97.

15. Disis ML, et al. HER-2/neu vaccine-primed autologous T-cell infusions for the treatment of advanced stage HER-2/neu expressing cancers. Cancer Immunol Immunother. 2014;63(2):101-109.

16. Mennuni C, et al. Efficient induction of T-cell responses to carcinoembryonic antigen by a heterologous prime-boost regimen using DNA and adenovirus vectors carrying a codon usage optimized cDNA. Int J Cancer. 2005;117(3):444-455.

17. Pierini S, et al. A Tumor Mitochondria Vaccine Protects against Experimental Renal Cell Carcinoma. J Immunol. 2015;195(8):4020-4027.

18. Parviz M, et al. Successful adoptive immunotherapy with vaccine-sensitized $\mathrm{T}$ cells, despite no effect with vaccination alone in a weakly immunogenic tumor model. Cancer Immunol Immunother. 2003;52(12):739-750.

19. Teshima T, Liu C, Lowler KP, Dranoff G, Ferrara JL. Donor leukocyte infusion from immunized donors increases tumor vaccine efficacy after allogeneic bone marrow transplantation. Cancer Res. 2002;62(3):796-800.

20. Joseph RW, et al. Impact of clinical and pathologic features on tumor-infiltrating lymphocyte expansion from surgically excised melanoma metastases for adoptive T-cell therapy. Clin Cancer Res. 2011;17(14):4882-4891.

21. Round JL, O'Connell RM, Mazmanian SK. Coordination of tolerogenic immune responses by the commensal microbiota. $J$ Autoimmun. 2010;34(3):J220-J225.

22. Ivanov II, et al. Induction of intestinal Th17 cells by segmented filamentous bacteria. Cell. 2009;139(3):485-498

23. Atarashi K, et al. Treg induction by a rationally selected mixture of Clostridia strains from the human microbiota. Nature. 2013;500(7461):232-236.

24. Round JL, Mazmanian SK. Inducible Foxp3+ regulatory T-cell development by a commensal bacterium of the intestinal microbiota. Proc Natl Acad Sci USA. 2010;107(27):12204-12209.

25. Rao VP, et al. Innate immune inflammatory response against enteric bacteria Helicobacter hepaticus induces mammary adenocarcinoma in mice. Cancer Res. 2006;66(15):7395-7400.

26. Lakritz JR, et al. Beneficial bacteria stimulate host immune cells to counteract dietary and genetic predisposition to mammary cancer in mice. Int J Cancer. 2014;135(3):529-540.

27. Vétizou M, et al. Anticancer immunotherapy by CTLA-4 blockade relies on the gut microbiota. Science. 2015;350(6264):1079-1084.

28. Sivan A, et al. Commensal Bifidobacterium promotes antitumor immunity and facilitates anti-PD-L1 efficacy. Science. 2015;350(6264):1084-1089.

29. Iida N, et al. Commensal bacteria control cancer response to therapy by modulating the tumor microenvironment. Science. 2013;342(6161):967-970.

30. Paulos CM, et al. Microbial translocation augments the function of adoptively transferred self/tumor-specific CD8+ T cells via TLR4 signaling. J Clin Invest. 2007;117(8):2197-2204.

31. Lanzavecchia A, Sallusto F. The instructive role of dendritic cells on T cell responses: lineages, plasticity and kinetics. Curr Opin Immunol. 2001;13(3):291-298.

32. Doulatov S, Notta F, Eppert K, Nguyen LT, Ohashi PS, Dick JE. Revised map of the human progenitor hierarchy shows the origin of macrophages and dendritic cells in early lymphoid development. Nat Immunol. 2010;11(7):585-593.

33. Kalinski P, Muthuswamy R, Urban J. Dendritic cells in cancer immunotherapy: vaccines and combination immunotherapies. 
Expert Rev Vaccines. 2013;12(3):285-295.

34. Veglia F, Gabrilovich DI. Dendritic cells in cancer: the role revisited. Curr Opin Immunol. 2017;45:43-51.

35. Wu HJ, Wu E. The role of gut microbiota in immune homeostasis and autoimmunity. Gut Microbes. 2012;3(1):4-14.

36. Ericsson AC, et al. Effects of vendor and genetic background on the composition of the fecal microbiota of inbred mice. PLoS ONE. 2015;10(2):e0116704.

37. Ji H, Chang EY, Lin KY, Kurman RJ, Pardoll DM, Wu TC. Antigen-specific immunotherapy for murine lung metastatic tumors expressing human papillomavirus type 16 E7 oncoprotein. Int J Cancer. 1998;78(1):41-45.

38. Restifo NP, Dudley ME, Rosenberg SA. Adoptive immunotherapy for cancer: harnessing the T cell response. Nat Rev Immunol. 2012;12(4):269-281.

39. Yan J, et al. Induction of antitumor immunity in vivo following delivery of a novel HPV-16 DNA vaccine encoding an E6/E7 fusion antigen. Vaccine. 2009;27(3):431-440.

40. Hansen $\mathrm{CH}$, et al. Patterns of early gut colonization shape future immune responses of the host. PLoS ONE. 2012;7(3):e34043

41. Rao S, Kupfer Y, Pagala M, Chapnick E, Tessler S. Systemic absorption of oral vancomycin in patients with Clostridium difficile infection. Scand J Infect Dis. 2011;43(5):386-388.

42. Fekety R. Guidelines for the diagnosis and management of Clostridium difficile-associated diarrhea and colitis. American College of Gastroenterology, Practice Parameters Committee. Am J Gastroenterol. 1997;92(5):739-750.

43. Hill DA, et al. Metagenomic analyses reveal antibiotic-induced temporal and spatial changes in intestinal microbiota with associated alterations in immune cell homeostasis. Mucosal Immunol. 2010;3(2):148-158.

44. Ivanov II, et al. Specific microbiota direct the differentiation of IL-17-producing T-helper cells in the mucosa of the small intestine. Cell Host Microbe. 2008;4(4):337-349.

45. Winter H, Hu HM, Urba WJ, Fox BA. Tumor regression after adoptive transfer of effector T cells is independent of perforin or Fas ligand (APO-1L/CD95L). J Immunol. 1999;163(8):4462-4472.

46. Gately MK. Interleukin-12: a recently discovered cytokine with potential for enhancing cell-mediated immune responses to tumors. Cancer Invest. 1993;11(4):500-506.

47. Mehrotra PT, Wu D, Crim JA, Mostowski HS, Siegel JP. Effects of IL-12 on the generation of cytotoxic activity in human CD8+ T lymphocytes. J Immunol. 1993;151(5):2444-2452.

48. Shortman K, Heath WR. The CD8+ dendritic cell subset. Immunol Rev. 2010;234(1):18-31.

49. Lee Y, et al. Therapeutic effects of ablative radiation on local tumor require CD8+ T cells: changing strategies for cancer treatment. Blood. 2009;114(3):589-595.

50. Schulz O, et al. CD40 triggering of heterodimeric IL-12 p70 production by dendritic cells in vivo requires a microbial priming signal. Immunity. 2000;13(4):453-462.

51. Bowers JS, et al. Dendritic Cells in Irradiated Mice Trigger the Functional Plasticity and Antitumor Activity of Adoptively Transferred Tc17 Cells via IL12 Signaling. Clin Cancer Res. 2015;21(11):2546-2557.

52. Karin M, Jobin C, Balkwill F. Chemotherapy, immunity and microbiota--a new triumvirate? Nat Med. 2014;20(2):126-127.

53. Viaud S, et al. The intestinal microbiota modulates the anticancer immune effects of cyclophosphamide. Science. 2013;342(6161):971-976.

54. Vétizou M, et al. Anticancer immunotherapy by CTLA-4 blockade relies on the gut microbiota. Science. 2015;350(6264):1079-1084

55. Chu H, Mazmanian SK. Innate immune recognition of the microbiota promotes host-microbial symbiosis. Nat Immunol. 2013;14(7):668-675.

56. Mazmanian SK, Round JL, Kasper DL. A microbial symbiosis factor prevents intestinal inflammatory disease. Nature. 2008;453(7195):620-625.

57. Cheng WF, et al. Tumor-specific immunity and antiangiogenesis generated by a DNA vaccine encoding calreticulin linked to a tumor antigen. J Clin Invest. 2001;108(5):669-678.

58. Kim TW, Lee JH, He L, Boyd DA, Hung CF, Wu TC. DNA vaccines employing intracellular targeting strategies and a strategy to prolong dendritic cell life generate a higher number of CD8+ memory $\mathrm{T}$ cells and better long-term antitumor effects compared with a DNA prime-vaccinia boost regimen. Hum Gene Ther. 2005;16(1):26-34.

59. Kim MS, Sin JI. Both antigen optimization and lysosomal targeting are required for enhanced anti-tumour protective immunity in a human papillomavirus E7-expressing animal tumour model. Immunology. 2005;116(2):255-266.

60. Lança T, Silva-Santos B. The split nature of tumor-infiltrating leukocytes: Implications for cancer surveillance and immunotherapy. Oncoimmunology. 2012;1(5):717-725.

61. Palucka K, Banchereau J. Cancer immunotherapy via dendritic cells. Nat Rev Cancer. 2012;12(4):265-277.

62. Segal JG, Lee NC, Tsung YL, Norton JA, Tsung K. The role of IFN-gamma in rejection of established tumors by IL-12 : source of production and target. Cancer Res. 2002;62(16):4696-4703.

63. Nastala CL, et al. Recombinant IL-12 administration induces tumor regression in association with IFN-gamma production. $J$ Immunol. 1994;153(4):1697-1706.

64. Fujiwara D, et al. Systemic control of plasmacytoid dendritic cells by CD8+ T cells and commensal microbiota. J Immunol. 2008;180(9):5843-5852.

65. Trinchieri G. Interleukin-12 and the regulation of innate resistance and adaptive immunity. Nat Rev Immunol. 2003;3(2):133-146.

66. Trinchieri G, Pflanz S, Kastelein RA. The IL-12 family of heterodimeric cytokines: new players in the regulation of T cell responses. Immunity. 2003;19(5):641-644.

67. Väyrynen JP, et al. The relationships between serum cytokine levels and tumor infiltrating immune cells and their clinical significance in colorectal cancer. Int J Cancer. 2016;139(1):112-121.

68. Lyakh L, Trinchieri G, Provezza L, Carra G, Gerosa F. Regulation of interleukin-12/interleukin-23 production and the T-helper 17 response in humans. Immunol Rev. 2008;226:112-131.

69. Xu M, Mizoguchi I, Morishima N, Chiba Y, Mizuguchi J, Yoshimoto T. Regulation of antitumor immune responses by the IL-12 family cytokines, IL-12, IL-23, and IL-27. Clin Dev Immunol. 2010;2010:832454.

70. Snyder A, Pamer E, Wolchok J. IMMUNOTHERAPY. Could microbial therapy boost cancer immunotherapy? Science. 2015;350(6264):1031-1032. 
71. Nadkarni MA, Martin FE, Jacques NA, Hunter N. Determination of bacterial load by real-time PCR using a broad-range (universal) probe and primers set. Microbiology (Reading, Engl). 2002;148(Pt 1):257-266.

72. Barman M, et al. Enteric salmonellosis disrupts the microbial ecology of the murine gastrointestinal tract. Infect Immun. 2008;76(3):907-915.

73. Caporaso JG, et al. QIIME allows analysis of high-throughput community sequencing data. Nat Methods. 2010;7(5):335-336.

74. Edgar RC. Search and clustering orders of magnitude faster than BLAST. Bioinformatics. 2010;26(19):2460-2461.

75. McDonald D, et al. An improved Greengenes taxonomy with explicit ranks for ecological and evolutionary analyses of bacteria and archaea. ISME J. 2012;6(3):610-618.

76. Caporaso JG, Bittinger K, Bushman FD, DeSantis TZ, Andersen GL, Knight R. PyNAST: a flexible tool for aligning sequences to a template alignment. Bioinformatics. 2010;26(2):266-267.

77. Price MN, Dehal PS, Arkin AP. FastTree 2--approximately maximum-likelihood trees for large alignments. PLoS ONE. 2010;5(3):e9490.

78. Lozupone C, Knight R. UniFrac: a new phylogenetic method for comparing microbial communities. Appl Environ Microbiol. 2005;71(12):8228-8235.

79. Anderson, Marti J. A new method for non-parametric multivariate analysis of variance. Austral Ecol. 2001;26:32-46. 